Check for updates

Cite this: Mater. Chem. Front. 2020, 4, 2548

Received 4th June 2020 ,

Accepted 6th July 2020

DOI: 10.1039/d0qm00376j

rsc.li/frontiers-materials

\section{Detection of biomarkers in body fluids using bioprobes based on aggregation-induced emission fluorogens}

\author{
Xinyi Zhang, ${ }^{a b}$ Bicheng Yao, ${ }^{c d}$ Qi Hu, ${ }^{a}$ Yuning Hong, (iD cd Angus Wallace, ${ }^{\text {ab }}$ \\ Karen Reynolds, ${ }^{a b}$ Carolyn Ramsey, ${ }^{a b}$ Anthony Maeder, ${ }^{a b}$ Richard Reed $^{a}$ and \\ Youhong Tang id *ab
}

\begin{abstract}
Body fluids (biofluids) are used as a means of examining various biomarkers of health and disease both as a means of rapid diagnosis and monitoring of chronic health conditions. Biomarker detection in noninvasively collected biofluids is considered an easy and rapid method in medical diagnostics. A novel group of bioprobes with aggregation-induced emission (AIE) properties is being developed for monitoring analytes in biofluids which could facilitate widespread use via commonly available technologies. This review describes the constituents and clinical biomarkers in urine, saliva, and sweat, and the role of currently developed AIE bioprobes that can quantitatively detect disease-related biomarkers in these biofluids. Several applications of AIE bioprobes, such as paper-based strips and POC devices, are currently under development, and offer potential to be realized simply by use with smartphone data capture and analysis and data cloud storage. These low-cost and highly reliable AIE bioprobe applications are beneficial for both early detection of disease and chronic disease management, especially in rural areas and developing countries and may facilitate greater involvement of health care consumers in managing their own care.
\end{abstract}

\section{Introduction}

Body fluids, or biofluids, including urine, saliva, sweat, tears, mucus, blood, cerebrospinal fluid, gastric juice, and semen contain complex mixtures of substances. ${ }^{1}$ The composition and level of these substances can reflect the health status of the human body. Biofluid samples can be collected by inserting a needle into the body and withdrawing, or via collection of such fluids into a container. The latter method is noninvasive and is applicable to biofluids that are secreted outside the body, such as urine, saliva, and sweat, which are considered useful sources for clinical tests. Collection of such samples is easy and rapid compared to the collection of blood, enabling the development of portable devices for screening disease, monitoring chronic disease, and assessing clinical results during treatment. Once

\footnotetext{
${ }^{a}$ Australia-China Science and Research Fund-Joint Research Centre on Personal Health Technologies, Flinders University, South Australia 5042, Australia. E-mail: youhong.tang@flinders.edu.au; Tel: +61-8-82012138

${ }^{b}$ Medical Device Research Institute, Flinders University, South Australia 5042, Australia

${ }^{c}$ Australia-China Science and Research Fund-Joint Research Centre on Personal Health Technologies, La Trobe University, Victoria 3086, Australia

${ }^{d}$ Department of Chemistry and Physics, La Trobe Institute for Molecular Science, La Trobe University, Victoria 3086, Australia
}

samples are obtained, tests that can be conducted include but are not limited to chemistry tests, microscopic examination, immunological tests, and nucleic acid-based tests. However, biofluid analysis presents several challenges, such as analyte complexity and the wide dynamic range of analyte abundance. ${ }^{2}$

Each biofluid has its own advantages and disadvantages due to its properties. The largest component of non-invasively collected biofluids such as urine, saliva and sweat is water. These biofluids have different levels of biomolecules respectively. The major components of urine are urea, chloride, and sodium and potassium salts. ${ }^{3}$ Saliva is the easiest obtained biofluid comparing to urine and sweat, and it includes a variety of electrolytes such as sodium, potassium, calcium, magnesium, bicarbonate, and phosphates. ${ }^{4}$ Applications of sweat are as a biofluid for clinical use are limited. Sodium and chloride are the primary electrolytes in sweat. Sweat also has amounts of minerals, lactic acid, and urea. ${ }^{5-7}$ Recent decades have seen an increased research focus on the analysis of non-invasively collected biofluids using bioprobes with high sensitivity and specificity, with the development of corresponding detection devices with low cost and high portability.

Fluorescent bioprobes have good sensitivity and selectivity for quantitative analysis of biomarkers in medical diagnostics. ${ }^{8}$ Most fluorophores are produced as nanoparticles/colloids or 
quantum dots only nanometers in size. For the use of such materials as fluorescent bioprobes, serious drawbacks remain, such as aggregation-caused quenching (ACQ) effect or significant toxicity issues due to the heavy metals involved. ACQ fluorophores often encounter fluorescence reduction or quenching, leading to a reduction in test sensitivity. ${ }^{9}$ This effect limits the scope of application of such materials as fluorescent bioprobes in both liquid and solid states.

In 2001, Tang et al. discovered that the light-emitting process of certain fluorogens can be constructed but not destroyed by molecular aggregation. The compound hexaphenylsilole (HPS) is non-luminescent when molecularly dissolved in good solvent (acetonitrile), and can be guided to emit light under aggregate formation. ${ }^{10}$ This novel phenomenon, known as 'aggregationinduced emission' (AIE), allows the use of dye solution of any concentration for bioassays and permits the development of biosensors using fluorogen aggregation to produce quantifiable light. ${ }^{10}$ The mechanism of AIE results from the restriction of intramolecular motion in the progress of aggregate formation. Therefore, the AIE phenomenon is usually observed in molecules that contain rotating or vibrating units, such as phenyl rings. Fluorogens with rotors experience lowfrequency motions in dilute solution, leading to fast nonradiative decay of the excited state. This process causes the fluorogens to be non-emissive in solution. In the aggregates, intermolecular steric interaction restricts the motions of the motors, encouraging opening of a radiative pathway for the fluorogens. ${ }^{11}$ Thanks to nearly 20 years of flourishing development in the AIE field, various AIE fluorogens (AIEgens) have been developed and their applications have been studied for valuable materials such as chemosensors, bioprobes, and emitters in the solid state. ${ }^{10}$ The new bioprobes show advanced features in respect of brightness, biocompatibility, and cellular retention. Moreover, the performance of AIE bioprobes can be further improved by particle formulation and surface functionalization. Another advantage is the conjugation of AIEgens and receptors producing light-up bioprobes for specific analyte monitoring. That enables direct visualization of the analytes and their processing in aqueous media. The nano-aggregated AIE bioprobes are organic versions of inorganic quantum dots (QDs) probes, with the added advantages that AIE nano-bioprobes are less cytotoxic but much brighter, if not at least as sensitive as inorganic QDS probes. ${ }^{10}$ Thiolated metal nanoclusters (NCs) are a class of novel luminescent materials in biomedical field due to their inherent biocompatibility and good luminescence properties. Highly photostable and long luminescence lifetime are observed in this AIE-type metal NCs. However, they do not have toxic heavy metals. Researchers recently synthesized a group of the AIE-type metal NCs for biomedical applications. ${ }^{12}$ In general, the merits of AIE provide a new opportunity for monitoring the levels of analytes in human body fluids. This review summarizes a group of novel fluorescent bioprobes based on the AIE concept that can be used to conduct quantitative analysis for biomarker detection in urine, saliva, and sweat.

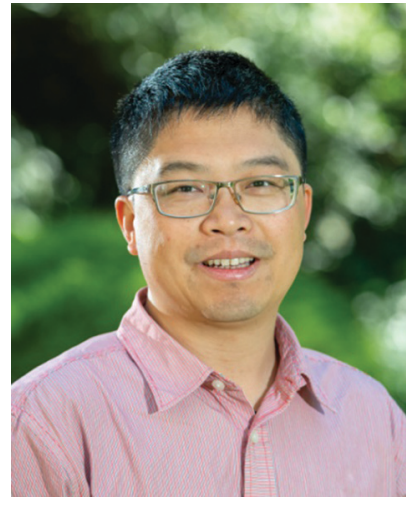

Youhong Tang
Dr Youhong Tang is a Professor and was an Australian Research Council-Discovery Early Career Researcher (ARC-DECRA) in Flinders University. Currently, he is the Deputy Director of International Laboratory for Health Technologies and Deputy Director of ACSRF-Joint Research Centre on Personal Health Technologies, a research leader in Institute for Nanoscale Science and Technology and a member of management committee of Medical Device Research Institute in Flinders University, Australia. He was elected as a Project Management Professional of Project Management Institute (PMI), US in 2008 and a Fellow of Royal Society of Chemistry (FRSC), UK in 2018. Dr Tang obtained his PhD degree in the Hong Kong University of Science and Technology (HKUST) in 2007 and moved to Flinders University in 2012 from the University of Sydney. He is a material science and engineering researcher with research interests mainly focused on (1) structure-processing-property relationship of polymeric (nano)materials; (2) biomaterials, biosensors and their devices with aggregation-induced emission features. 


\section{Urine}

Urine is a body fluid that can provide substantial information about individual health and disease. Urine analysis is a noninvasive means to evaluate the functional status of many organs, especially the kidney. It can also be used to monitor body homeostasis and some metabolic disease processes due to its capability of plasma ultrafiltration. ${ }^{3}$ Kidneys produce 600 to $1800 \mathrm{~mL}$ urine each day, of which the largest component is water, which is comprised of $95 \%$ urine, ${ }^{13}$ the other major components being urea, chloride, and sodium and potassium salts. Remaining substances are phosphate, sulfate, creatinine, and uric acid. The urine of a normal healthy individual does not contain significant amounts of glucose, bicarbonate, or albumin, which are normally completely reabsorbed by the kidney. ${ }^{3}$ Normal values for urine chemical components are shown in Table 1. Therefore, urine can be used to test metabolic disease, kidney physiological process, infection of the urinary tract, drugs, toxicology, etc. ${ }^{13}$ Urinalysis involves three parts: visual examination, chemical examination, and microscopic examination. Microscopic examination can monitor the counts and types of cells, casts, crystals, bacteria, and mucus. Reagent strips are frequently used as convenient methods used for routine screening of 10 medically important components such as proteins, glucose, ketones, and bilirubin (see Table 1). However, test strips can provide false negative and false positive results, giving misleading information. ${ }^{13}$ Many researchers develop optical sensors for biomolecules in urine. The biosensors could integrate with portable device for fast and accurate urine detection, but there are still some challenges such as strong interference by other biomolecules.

In this section, we introduce examples of AIEgens used in urinary biomarker detection. These biomarkers include albumin, creatinine, citrate, hyaluronidase, pyrophosphate (PPi), dopamine, and heavy metal ions, etc.

\subsection{Albumin}

Human serum albumin (HSA) can reach $35-50 \mathrm{~g} \mathrm{~L}^{-1}$ in blood plasma but is less than $30 \mathrm{mg} \mathrm{L}^{-1}$ in urine because of renal filtration. Hence, albuminuria can be used as a diagnostic index for chronic kidney disease. ${ }^{14}$ There are many reports of AIEgen designed for detecting albuminuria. The AIEgens

Table 1 Normal values for urine chemical components when measured by reagent test strips ${ }^{13}$

\begin{tabular}{ll}
\hline Analyte & Normal $^{a}$ \\
\hline Glucose & Negative to trace \\
Bilirubin & Negative \\
Ketone & Negative \\
Specific gravity & $1.005-1.030$ \\
Blood & Negative \\
pH & $5.0-6.0$ \\
Protein & Negative to trace \\
Urobilinogen & $0.2-1.0 \mathrm{mg} \mathrm{dL}^{-1}$ \\
Nitrite & Negative \\
Leukocytes & Negative \\
$a$ Range that is found in most normal individuals.
\end{tabular}

exhibit changes in emission features, such as intensity, wavelength, or lifetime, that can be used to signal the binding process of AIEgens to albumin and thus reflect the level of albumin in the solution.

HSA is a globular protein with three $\alpha$-helical domains (I-III), each of which is further divided into two subdomains (A and B), as shown in Scheme 1. Two major ligand-binding sites (site 1 and site 2) are located in the hydrophobic cavities of subdomains IIA and IIIA respectively. ${ }^{15}$ These two structurally selective binding sites are responsible for the specific recognition of ligands. In these binding sites, different binding affinities and interaction forces exist to bind molecules that can perform different functions. Thus, binding studies enable prediction of the molecular mechanisms of ligand-selective interactions and improvement of bioprobe design for achieving good selectivity and sensitivity. ${ }^{16}$ Base on this, scientists develop a series of AIE bioprobes to evaluate albumin (Table 2).

Probe BSPOTPE (sodium 1,2-bis[4-(3-sulfonatopropoxyl)phenyl]-1,2-diphenylethene), first reported by Hong et al. in 2010, has been demonstrated having good performance on monitoring albumin with a limit of detection (LOD) as low as $1 \mathrm{nM}$ (Fig. 1). ${ }^{15}$ This probe is amphiphilic, has excellent watersolubility and shows an excellent preference to hydrophobic cavities surrounded by subdomains IIIA, IB and IIA. HSA's hydrophobic regions can accommodate insoluble endogenous compounds, which may explain why the BSPOTPE presents good selectivity toward albumin proteins. Additionally, BSPOTPE can detect HSA in a linear range of 0-100 nM indicating its high sensitivity towards HSA. The study of Hong and coworkers also showed that this bioassay is tolerant of the interfering substances in the artificial urine. ${ }^{15}$

Gabr and Pigge reported that an organometallic bioprobe with AIE properties, TAE-Re(I) that was prepared from bis(pyridyl)- and bis(quinolyl) tetraarylethylene (TAE) ligands, presented specific binding to site 2 of HSA. ${ }^{17}$ The binding process resulted in a blue shifted emission $(\sim 95 \mathrm{~nm})$ with a

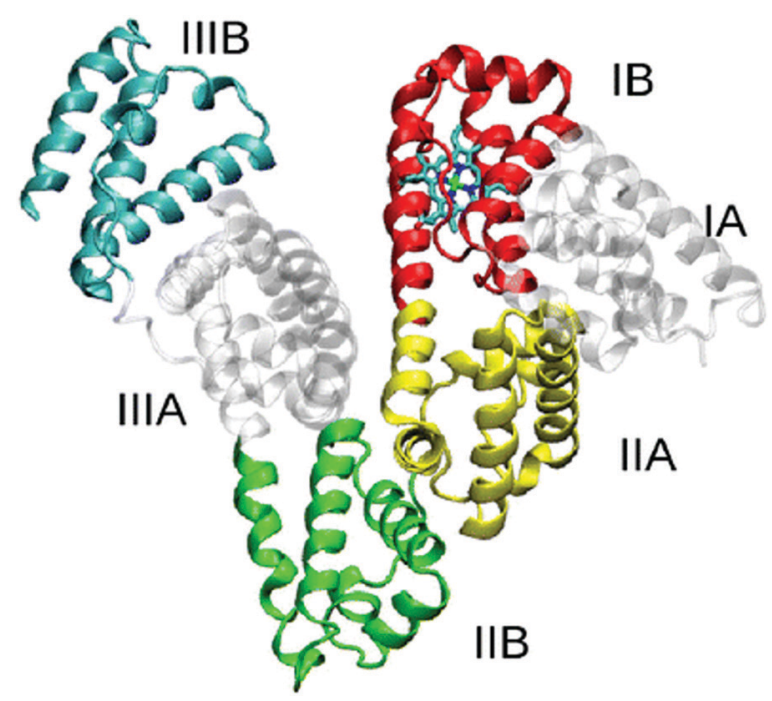

Scheme 1 Structure of HSA (RSCB POB: 1AO6). Adapted from Wikipedia. 
Table 2 AlEgens for BSA or HSA detection

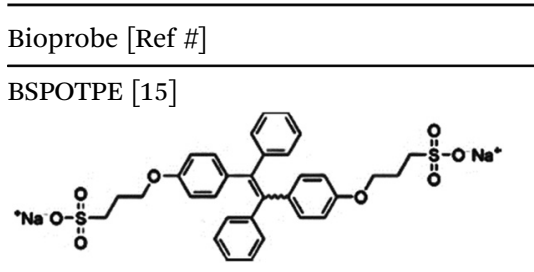

TAE-Re(I) [17]

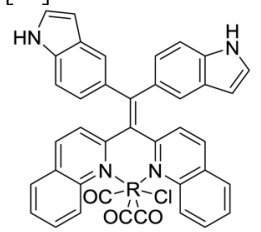

M2 [18]<smiles>O=[N+]([O-])CCCOc1ccc(C(=C(c2ccccc2)c2ccccc2)c2ccc(O)cc2)cc1</smiles>

L1 [8]<smiles>O=C(Nc1ccc2cc3ccccc3cc2c1)Nc1cccc2ccccc12</smiles>

BQN2 [19]<smiles></smiles>

Nanoprobe 7 [20]<smiles>Br[n+]1cccc2ccccc21</smiles>

Compound 1 [21]<smiles>CN(C)CCCCCCCCn1c2ccc(-c3nc4ccccc4[nH]3)cc2c2cc(-c3nc4ccccc4[nH]3)ccc21</smiles>

TPE-4TA [22]<smiles></smiles>

$396 / 509 \mathrm{~nm}$

NA

Linear range

0-100 nM

LOD
$1 \mathrm{nM}$

$1 \mathrm{nM}$ Test media

$350 / 475 \mathrm{~nm}$

$330 / 476 \mathrm{~nm}$

$380 / 438 \mathrm{~nm}$

NA

NA

$0-100 \mu \mathrm{g} \mathrm{mL}{ }^{-1}$

$0-0.8 \mathrm{mg} \mathrm{mL} \mathrm{m}^{-1}$

$5 \mu \mathrm{g} \mathrm{mL}^{-1}$

NA

$380 / 480 \mathrm{~nm}$

$0-15 \mu \mathrm{M}$

$350 / 454 \mathrm{~nm}$

$$
\text { 0-80 mg L }{ }^{-1}
$$

$0.27 \mathrm{mg} \mathrm{L}^{-1}$

Human urine
Artificial urine

Artificial urine
$0.14 \mu \mathrm{M}\left(9.3 \mathrm{mg} \mathrm{L}^{-1}\right)$

NA
NA

Artificial urine<smiles>C1CCCCC1</smiles> 
Table 2 (continued)

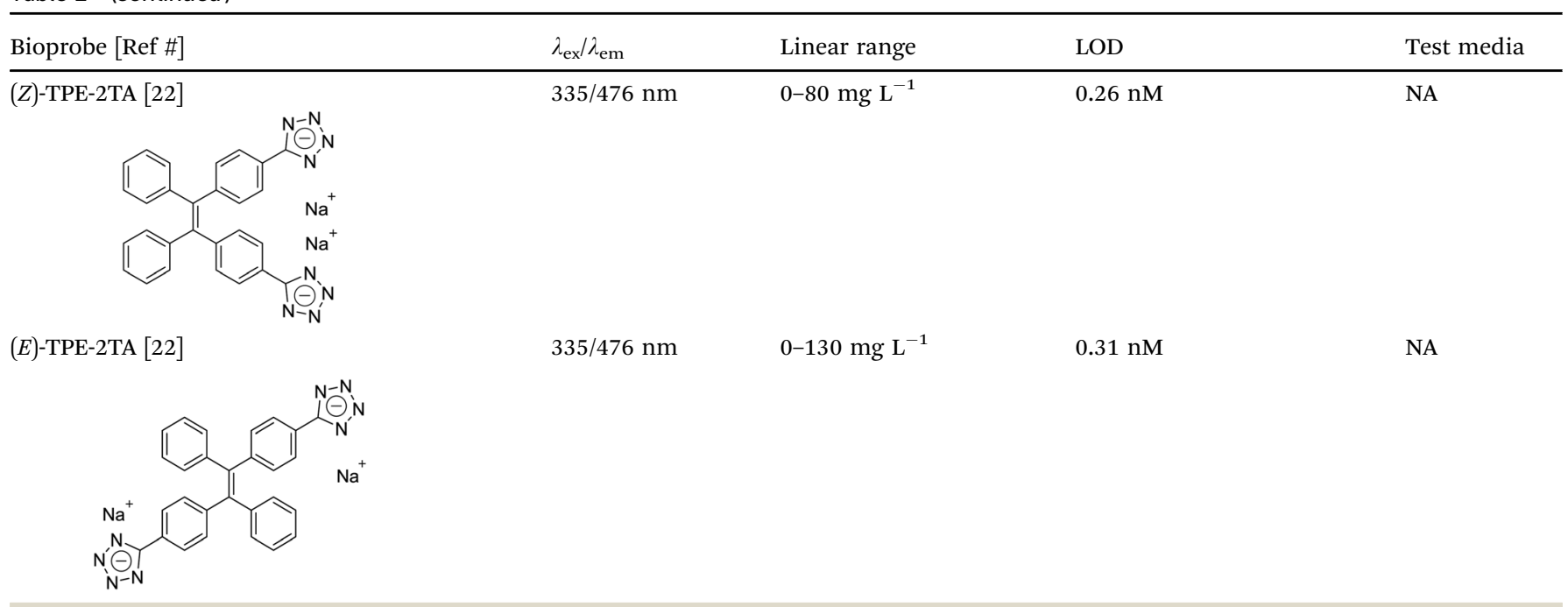
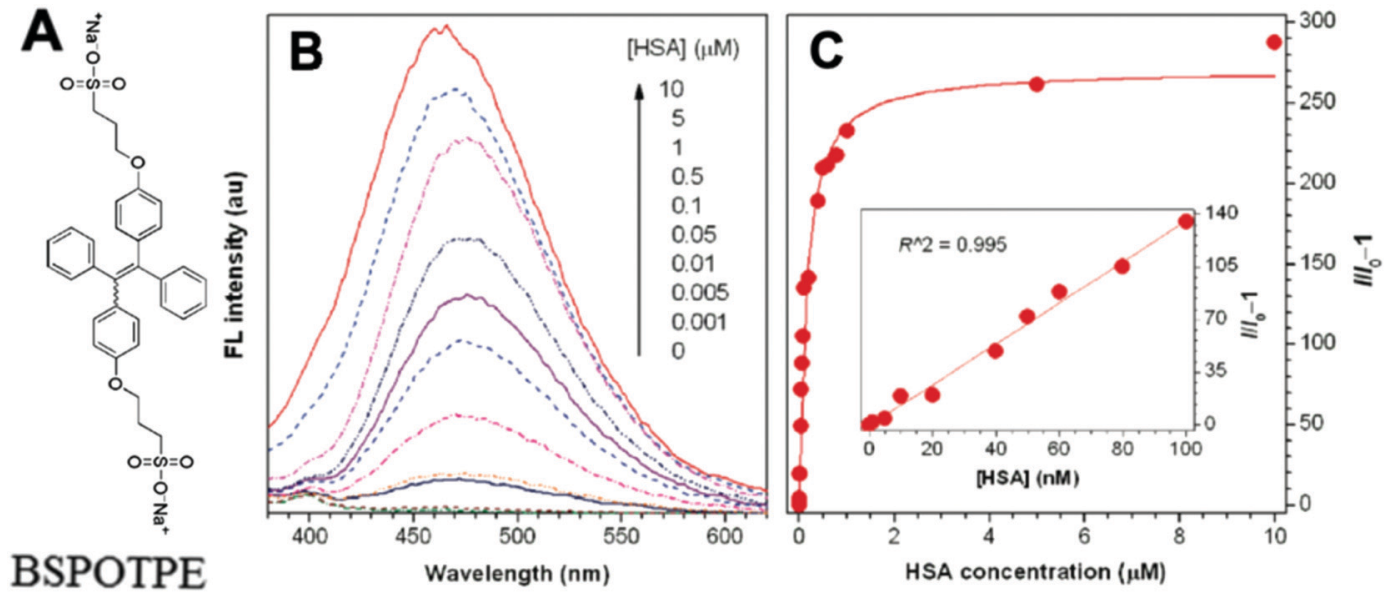

Fig. 1 (A) Chemical structure of BSPOTPE. (B) FL spectra of BSPOTPE in the PBS buffer containing different amounts of HSA. (C) Change in the FL intensity at $475 \mathrm{~nm}$ with HSA concentration: $I_{0}=\mathrm{FL}$ intensity in the absence of HSA. Inset: Linear region of the binding isotherm of BSPOTPE to HSA. [BSPOTPE] $=5 \mu \mathrm{M} ; \lambda_{\mathrm{ex}}=350 \mathrm{~nm} .{ }^{15}$ Adapted with permission from ref. 15. Copyright 2010 American Chemical Society

25-fold enhancement in fluorescence intensity, where the excitation wavelength is $396 \mathrm{~nm}$ and the emission wavelength is $509 \mathrm{~nm}$. The detectable limit of HSA is $20 \mu \mathrm{M}$. The authors described that the folded structure of HSA is crucial for increasing the emission intensity of this bioprobe that was bonded to a hydrophobic binding site in the albumin. Additionally, the reaction of this AIEgen is not interfered by artificial urine environment, indicating compatibility in human urine. The researchers also reported that none of biomolecules were found to interfere with emission of this bioprobe interacting with HSA. In 2017, Tong et al. reported the design of some AIEgens to detect bovine serum albumin (BSA) with a structure similar to that of HSA. As shown in Fig. 2, a tetraphenylethene (TPE) derivative (M1) became highly fluorescent when combined with BSA and HSA. Tong et al. altered the molecular design of M1 by replacing two quaternary ammonium cations with two sulphonate anions (M2). M2 could detect BSA concentration as low as $500 \mathrm{ng} \mathrm{mL}^{-1}$ with a linear range of 0-100 $\mu \mathrm{g} \mathrm{mL} \mathrm{mL}^{-1}$. Probe M3 with a TPE core and two pyridinium moieties could emit red fluorescence, which had better performance than M1 and M2. Because of their AIE properties, both M2 and M3 showed emission enhancement when bound to the hydrophobic cavity of BSA, which is at the subdomain IIA. Moreover, the authors also concluded that hydrophobic interaction was the driving force of binding process, regardless of the negative or positive charges carried by probes. The detection of BSA using M2/M3 was not impaired by the bioelectrolytes present in artificial urine. ${ }^{18}$

Halder $e$ al. developed the AIE bioprobe L1 that enhances light intensity by binding with HSA through electrostatic interactions. Its detection limit is $5 \mu \mathrm{g} \mathrm{mL}{ }^{-1}$. Moreover, L1 has specific selectivity towards BSA/HSA and can be used as an efficient real-time bioprobe for monitoring HSA, which is attributed to the site-specific binding to the domains IIA and 
A

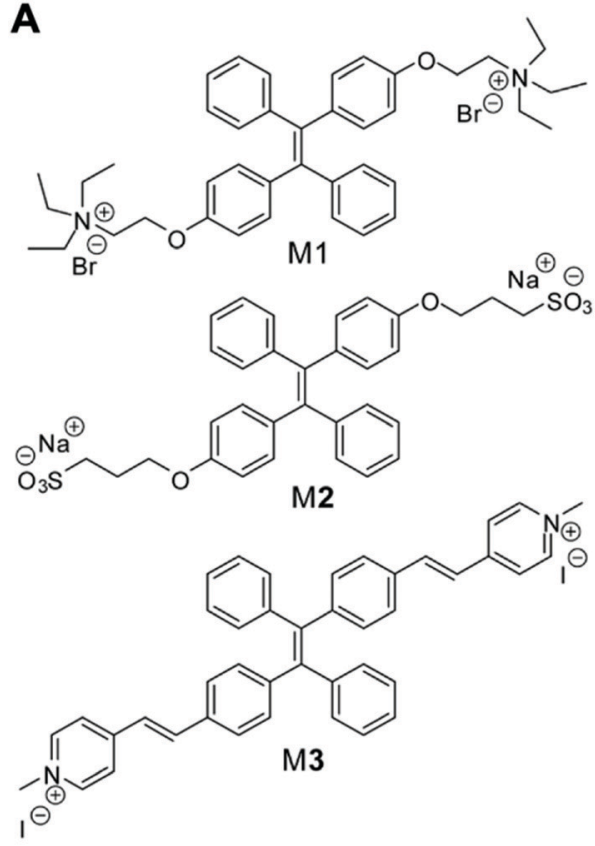

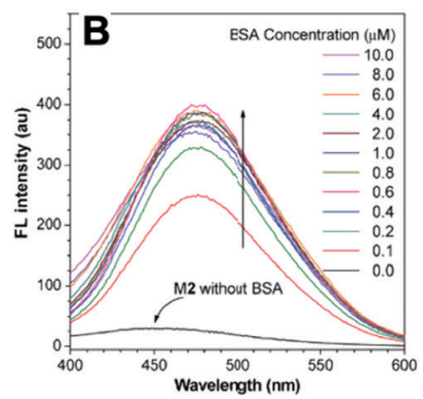
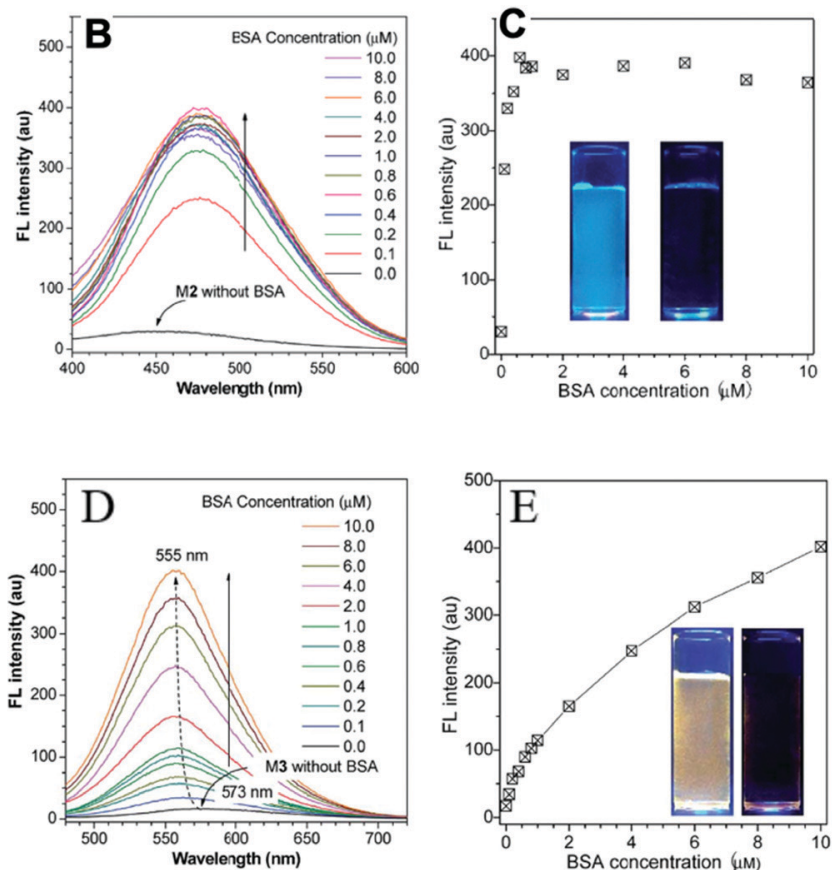

Fig. 2 (A) Chemical structures of the AlE-active fluorescent probes. (B) Fluorescence (FL) spectra of M2 in aqueous phosphate buffer solution (pH 7.4) with different concentrations of BSA. (C) Variation of FL peak intensity with BSA concentration, in which the data are extracted from the spectra shown in (B) at the emission wavelength of $459 \mathrm{~nm}$ for the sample without BSA and $476 \mathrm{~nm}$ for the other samples. Excitation wavelength ( $\lambda_{\text {ex }}$ ): $330 \mathrm{~nm}$; $\mathrm{M2}$ concentration ([M2]): $1 \mu \mathrm{M}$. Inset in (C): Photographs showing the emission of M2 in buffer solution ( $\mathrm{pH}$ 7.4) with and without BSA taken under illumination with $365 \mathrm{~nm}$ UV-light; (D) FL spectra of M3 in aqueous phosphate buffer solution (pH 7.4) with different concentrations of BSA; (E) variation of FL peak intensity with BSA concentration, in which the data are extracted from the spectra shown in (D). Excitation wavelength $\left(\lambda_{\text {ex }}\right): 395 \mathrm{~nm}$; [M3] $=1 \mu \mathrm{M}$. Inset in (E): Photographs showing the emission of $\mathrm{M} 3$ in buffer solution $(\mathrm{pH} 7.4)$ with and without BSA taken under illumination with $365 \mathrm{~nm}$ UV-light. Adapted with permission from ref. 18. Copyright (C) 2017 Royal Society of Chemistry.

IB of HSA. Moreover, L1 shows efficient clinical analysis in artificial urine samples. ${ }^{8}$ Singh et al. reported a boron dipyrromethene (BODIPY) based probe BODIPY(BQN2). The amphiphilic behavior of $\mathrm{BQN} 2$ and HSA enables both to interact through hydrophobic as well as H-bonding interactions. ${ }^{19}$ A bola-type nanoprobe 7 with AIE property was showed to be bonded to site 1 of HSA via multiple noncovalent interactions. The resultant restriction of intramolecular rotation of 7 in the hydrophobic cavity of HSA results in fluorescence emission. The detection limit of 7 was determined to be $0.14 \mu \mathrm{M}\left(9.3 \mathrm{mg} \mathrm{L}^{-1}\right)$. However, this probe was successfully applied in human serum samples only, rather than in urine. It has a high opportunity to indicate the compatibility in human urine. Furthermore, no fluorescence change was observed when other proteins and biomolecules were added. This observation showed that the nanoprobe 7 displayed high selectivity for HSA. ${ }^{20}$

Dey et al. showed that a bioprobe 'compound 1' could evaluate albumin content in human urine. Compound $\mathbf{1}$ is a unique example of a ratiometric sensor of HSA through reversible noncovalent interactions. The linear ratiometric response for HSA is $0-80 \mathrm{mg} \mathrm{L}^{-1}$ and the detection limit is $0.27 \mathrm{mg} \mathrm{L}^{-1}$. However, the urine samples used in that work had to be diluted 20 -fold. The selectivity investigation of compound $\mathbf{1}$ in the presence of various biologically ionic analytes, neutral compounds, and thiol-containing amino acids showed that only HSA induced an increase in fluorescent emission. ${ }^{21}$ In 2019, Tu et al. designed a series of specific and quantitative tetrazolate-functionalized AIEgens for albumin detection, namely TPE-4TA, (Z)-TPE-2TA, and $(E)$-TPE-2TA. Fluorescence assay showed that the minimum detectable HSA concentration was in the range of $0.21-0.31 \mathrm{nM}$. Multivalent tetrazolate-lysine interactions led to the binding of these probes, thus limiting their molecular motion. Further study showed that the main forces to trap the bioprobes in the albumin were enthalpically favored polar interactions including hydrogen bonds and coulombic forces. As shown in Fig. 3, TPE-4TA was shown to have the best performance, exhibiting a good linear dynamic range $\left(0.02-2500 \mathrm{mg} \mathrm{L}^{-1}\right)$ with a detection limit of $0.21 \mathrm{nM}$. Selectivity testing showed that TPE-4TA had no obvious response to proteins with isoelectric points ranging from 1 to 10 , such as hemoglobin, pepsin, and lysozyme. This study also used human urine samples for clinical diagnosis, indicating satisfactory accuracy and linearity. ${ }^{22}$

\subsection{Creatinine}

Creatinine is a waste product produced by muscle from the breakdown of a creatine which is used as an energy source for muscles. Creatinine is filtered by the kidney and its level in biological fluids is measured to evaluate renal function and muscle damage. ${ }^{23}$ The creatinine concentration in serum is between 35 and $40 \mu \mathrm{M}$ under a normal physiological status. Both increasing and decreasing concentrations can indicate diseases such as renal failure or muscular dystrophy. ${ }^{23}$ 

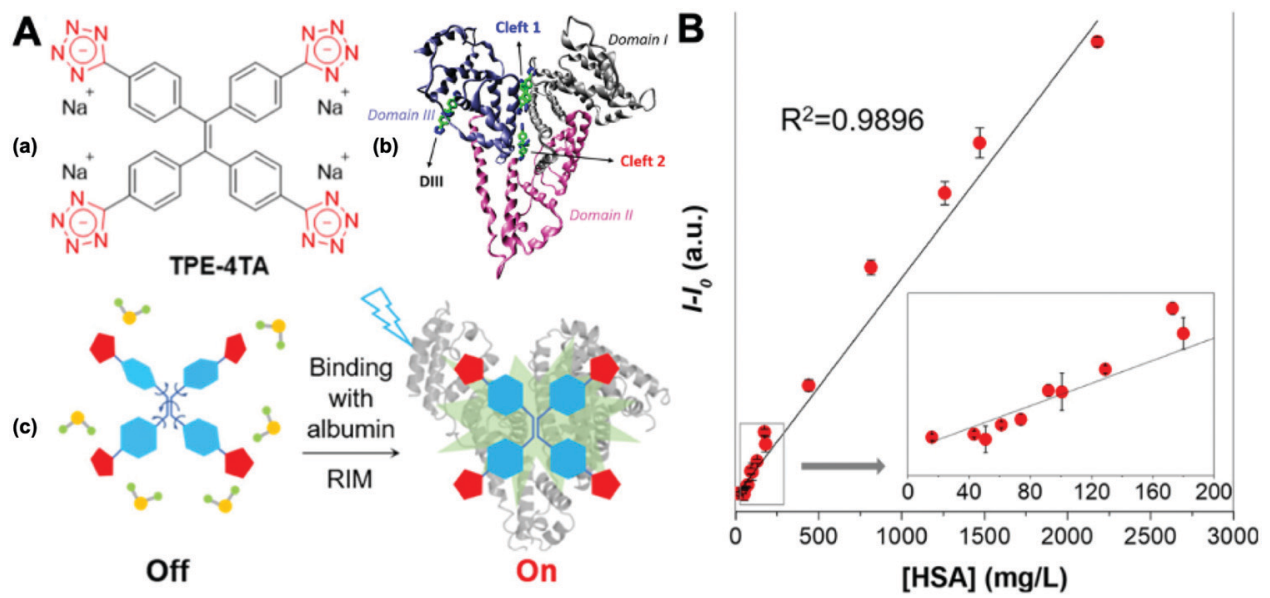

Fig. 3 (A) (a) Structure of TPE-4TA; (b) tetrazolate-tagged TPE probes for the albumin protein; (c) schematic illustration of the fluorescence switch in aqueous solutions and when binding to albumin. (B) Plot of albumin concentration in human urine samples by a turbidimetric inhibition immunoassay method ( $X$-axis) vs. the corresponding fluorescence intensity at $490 \mathrm{~nm}$ by fluorescent assay using TPE-4TA ( $Y$-axis). $I_{0}$ equals to the fluorescence intensity at $490 \mathrm{~nm}$ of urine blanks in the absence of the probe. Five repeats were used for each sample. Adapted with permission from ref. 22 . Copyright 2019 American Chemical Society.

A TPE salt IDATPE (iminodiacetic acid functionalized TPE) with the ability to detect creatinine was reported by our group in 2017 (Fig. 4). ${ }^{23}$ The excitation and emission wavelengths of IDATPE are $262 \mathrm{~nm}$ and $375 \mathrm{~nm}$, respectively. With the addition of creatinine, IDATPE coordinates easily with it through hydrogen bonds between the $-\mathrm{NH}$ group of creatinine and the $\mathrm{COO}^{-}$group of IDATPE. As a result, a large group is attached to the TPE core and restricts the rotation of the phenyl rings, resulting in a change in luminescent intensity. The emission of IDATPE increases in creatinine concentrations up to $100 \mu \mathrm{M}$ in artificial urine. ${ }^{23}$

Recently, Jalili and Khataee designed a fluorescent probe, based on glutathione-capped copper nanoclusters (CuNCs), for quantifiably detecting creatinine. The mechanism of this process is that when CuNCs meet $\mathrm{Al}^{3+}$ ions, the probe aggregates through glutathione- $\mathrm{Al}^{3+}$ interaction, inducing the enhancement of fluorescence emission. Furthermore, those CuNC aggregates are quenched by the addition of creatinine. The linear relationship between quenched fluorescent intensity and creatinine concentration is in the range of $2.5-34 \mu \mathrm{g} \mathrm{\textrm {L } ^ { - 1 }}$, and the lowest detectable concentration of creatinine can reach $0.63 \mu \mathrm{g} \mathrm{L} \mathrm{L}^{-1}$. The best light intensity is obtained at excitation and emission peaks of $360 / 585 \mathrm{~nm}$. Furthermore, the investigation of selectivity indicated that the levels of tested interferents were below their tolerable concentrations or could be decreased by dilution, showing the good selectivity of CuNCs. This probe was successfully used in human serum but has not been tested in human urine. ${ }^{24}$ There are not so many AIEgens for creatinine detection. It is necessary to do the urine test of this AIEgen for its future revision and application.

\subsection{Citrate}

Citrate is an organic tricarboxylate and is one of the crucial metabolites in the Krebs cycle for aerobic cells. Citrate levels in urine are related to several diseases, including kidney dysfunction and prostate cancer. ${ }^{25}$ A new turn-on fluorescent bioprobe TPE-Py was synthesized for the detection of citrate, caused by hydrogen bonding and electrostatic interactions. This bioprobe demonstrated good selectivity and sensitivity, with a low detection limit of $0.1 \mu \mathrm{M}$. Moreover, the test in artificial urine
A

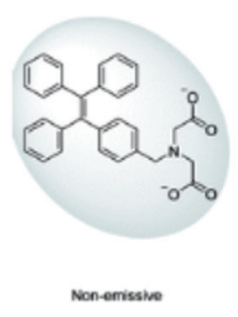

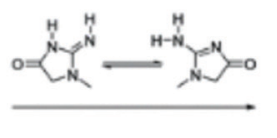

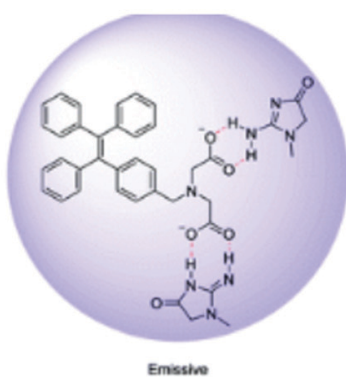

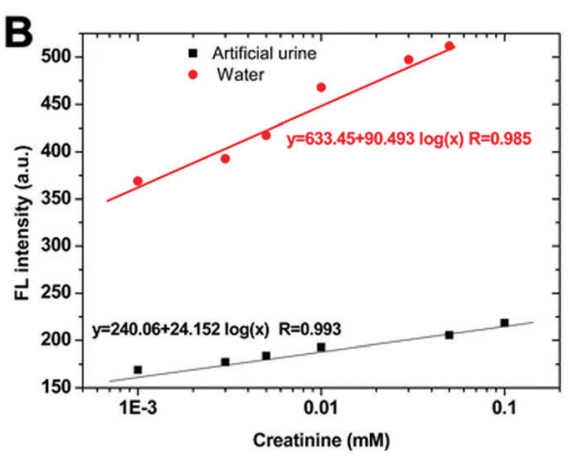

Fig. 4 (A) Schematic illustration of the possible mechanism of aggregation of IDATPE in the presence of creatinine. (B) Linear region in water and artificial urine. $\lambda_{\mathrm{ex}}=262 \mathrm{~nm}$; [IDATPE] $=40 \mu \mathrm{M}$; measured at $t=5 \mathrm{~min}$. Adapted with permission from ref. 23. Copyright 2017 Royal Society of Chemistry. 

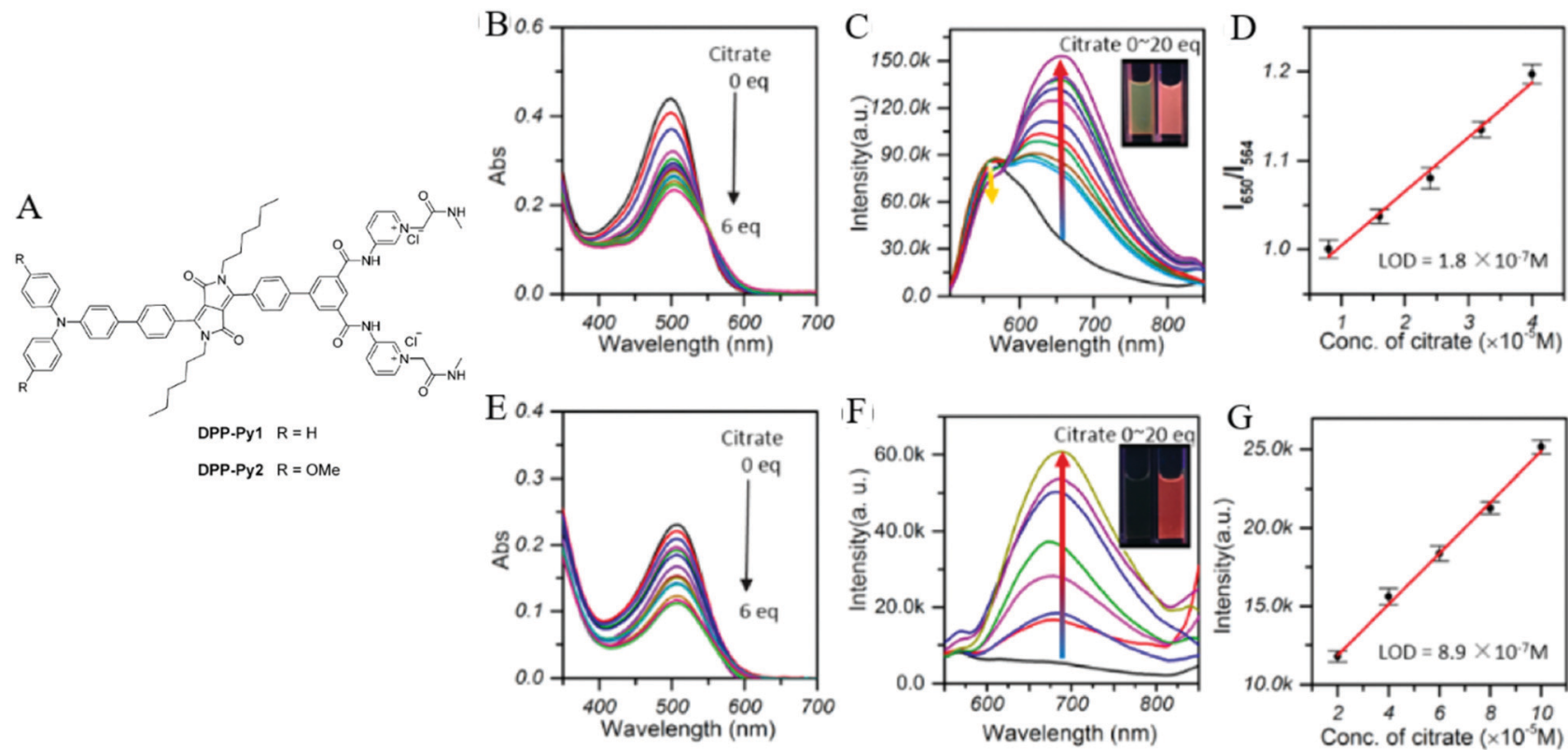

Fig. 5 (A) Structure of the target DPP-Py1 and DPP-Py2. Titration absorption spectra of (B) DPP-Py1 $\left(4 \times 10^{-5} \mathrm{M}\right)$ and (E) DPP-Py2 $\left(4 \times 10^{-5} \mathrm{M}\right)$ in HEPES buffer (10 mM, 70\% DMSO, pH 7.4) upon addition of 0.0-6.0 equivalent of citrate. Titration fluorescence spectra of (C) DPP-Py1 (4 $\left.\times 10^{-5} \mathrm{M}\right)$ and (F) DPP-Py2 in HEPES buffer (10 mM, 70\% DMSO, pH 7.4) upon addition of 0.0-20.0 equivalent of citrate. (D) Plots of the fluorescence intensity (ratio of $\left.I_{652} / I_{564}\right)$ as a function of citrate concentration for determination of the LOD of DPP-Py1. (G) Plots of the fluorescence intensity (values of emission maxima) as a function of citrate concentration for determination of the LOD of DPP-Py2. Inset (C): Photographs of DPP-Py1 solution before and after addition of citrate under a UV lamp with $365 \mathrm{~nm}$ incident light. Inset (F): Photographs of DPP-Py2 solution before and after addition of citrate under a UV lamp with $365 \mathrm{~nm}$ incident light. Adapted with permission from ref. 25. Copyright 2016 American Chemical Society.

showed that TPE-Py was capable of citrate quantification in real biological samples. ${ }^{26}$ Hang et al. reported two new diketopyrrolopyrrole-based fluorescent chemosensors, DPP-Py1 and DPP-Py2 (Fig. 5), that used symmetrical diamides to select and detect citrate in the near-infrared region. DPP-Py1 has good sensitivity with a low detection limit of $0.18 \mu \mathrm{M}$; the LOD of DPP-Py2 is $0.89 \mu \mathrm{M}$. In examination of their selectivity, it was found that other interferents did not induce any remarkable change of fluorescence. Both DPP-Py1 and DPP-Py2 had good selectivity for citrate among other analytes. Moreover, the researchers also applied these sensors in the quantification of citrate in artificial urine samples. The result presented good recovery values for using both probes. ${ }^{25} \mathrm{Jiang}$ et al. developed a highly selective fluorescent sensor, DTPA-TPY-Zn, for citrate detection. They integrated an AIE motif DTPA-TPY with $\mathrm{Zn}^{2+}$, producing DTPA-TPY-Zn with the relatively low detection limit of $0.35 \mu \mathrm{M}$. This probe also showed a high signal-to-noise ratio and excellent selectivity against citrate. The high recovery rate in artificial urine confirmed the accuracy and reliability of using DTPA-TPY-Zn for citrate quantification. ${ }^{27}$

\subsection{Hyaluronidase (HAase)}

HAase is an enzyme that plays an important role for degrading hyaluronic acid (HA) in the process of cancer cell metastasis. It is also relevant to many physiological and pathological processes, including inflammation, wound healing, and overexpression in certain cancers such as bladder cancer. ${ }^{28}$ HAase is reported to be urinary marker for early diagnosis of bladder cancer. ${ }^{29} \mathrm{Li}$ et al. synthesized a new AIE molecule
TPE- $4 \mathrm{~N}^{+}$, as shown in Fig. 6 . This probe gave rise to electrostatic adsorption towards HA, forming nanoparticles TPE-HA and leading to a fluorescent emission recovery in the yellow-green region at $536 \mathrm{~nm}$. When HAase was added into TPE-HA solution, the fluorescence was suppressed. Hence, TPE-HA can be used as a nanochemosensor for HAase detection in urine specimens. The detection limit was determined to be $0.02 \mathrm{U} \mathrm{mL}^{-1}$. The selectivity of TPE-HA for HAase was examined by checking fluorescence responses of TPE-HA to 13 other kinds of representative species. Only the addition of HAase induced a significant decrease in the emissive spectra. In a human urine test, samples spiked with fixed HAase concentrations were investigated. The average recoveries of HAase were in the range of $97-108 \%$ for all the spiked samples and a low relative standard deviation (0.95-1.08\%) was achieved. ${ }^{28}$

Xie et al. also reported a novel system based on the AIE and ACQ effects to detect HAase (Fig. 7). They developed AN- $\mathrm{N}^{+}$and TPE- $2 \mathrm{~N}^{+}$for sensing HAase through using unmodified HA as the nanoparticle scaffold and the substrate for enzymatic reaction. These two positively charged compounds were attached with a negatively charged HA, inducing the aggregation of the compounds as well as the formation of nanoparticles as a result of electrostatic complexation, with TPE- $2 \mathrm{~N}^{+}$acting as the cross-linking agent. The aggregation resulted in the quenching of the emission of $\mathrm{AN}^{-\mathrm{N}^{+}}$due to the ACQ effect, as well as the fluorescence enhancement of TPE-2 $\mathrm{N}^{+}$due to the AIE effect. In the presence of HAase, the enzymatic reaction caused the degradation of HA and induced disassembly of the nanoparticles. Hence, the emission of AN-N ${ }^{+}$was restored and that of TPE-2N ${ }^{+}$ 


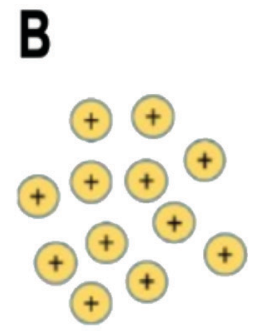

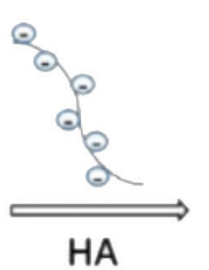

TPE $-4 \mathrm{~N}^{+}$
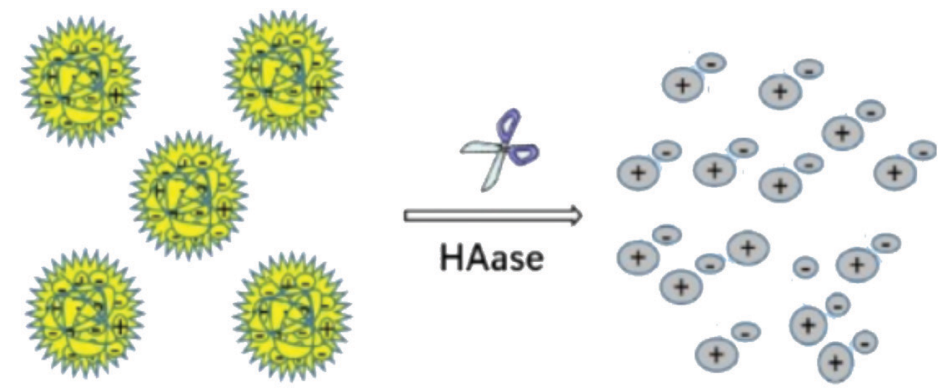

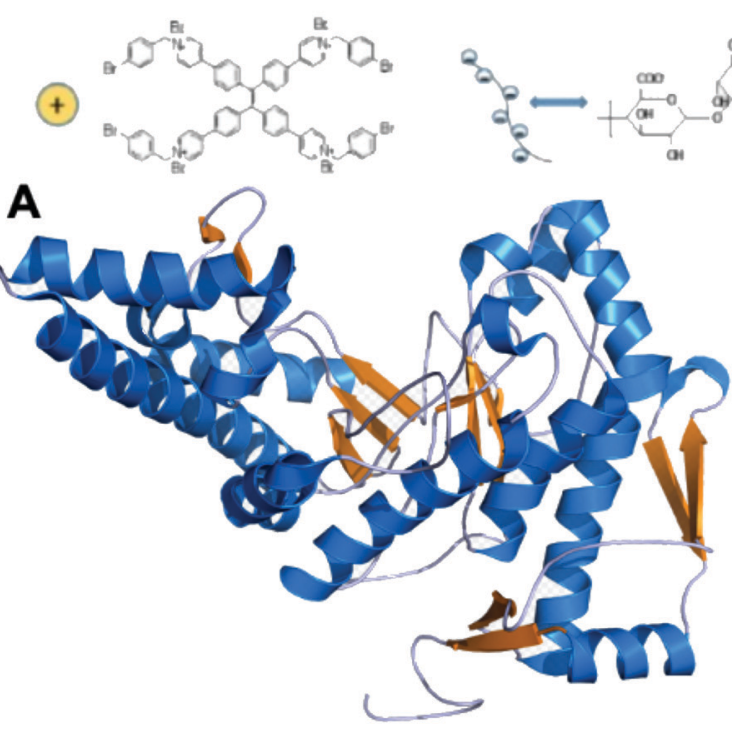

TPE-HA
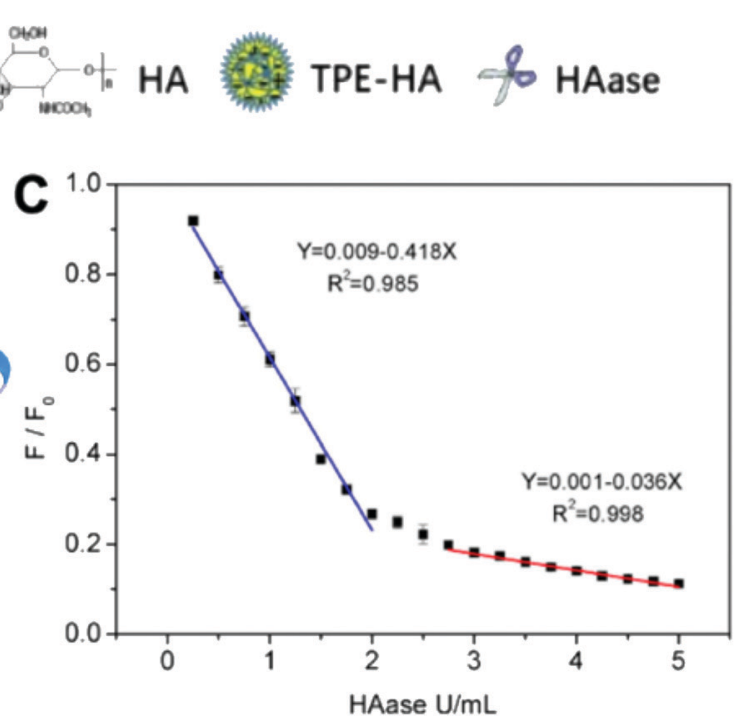

Fig. 6 (A) Structure of HAase. (B) Schematic illustration of TPE-HA based fluorescent nanoprobe for detection of HAase. (C) Plot of the relative intensity at $536 \mathrm{~nm}\left(F / F_{0}\right)$ against various HAase concentrations (from 0 to $\left.5 \mathrm{U} \mathrm{mL}^{-1}\right)\left(F_{0}\right.$ means initial emission intensity of TPE-HA (TPE-4N $1 \mu$ M, HA $0.15 \mu \mathrm{g} \mathrm{mL}^{-1}$ ) in aqueous solution, $F$ means emission intensity of TPE-HA in the presence of different concentrations of HAase), (equation for blue line: $Y$ refers to $F / F_{0}, X$ refers to concentration of HAase (ranging from 0.05 to $2 \mathrm{U} \mathrm{mL}{ }^{-1}$ ); equation for red line: $Y$ refers to $F / F_{0}, X$ refers to concentration of HAase (ranging from 2.75 to $\left.5 \mathrm{U} \mathrm{mL}^{-1}\right)$ ). All data represent mean $\pm \mathrm{SD}$ over three separate measurements. (A) Adapted from Wikipedia and (B and $\mathrm{C}$ ) Adapted with permission from ref. 28. Copyright 2018 Elsevier B.V.

was suppressed. This fluorescence change enabled the system to function as a robust ratiometric biosensor for HAase. The ratio of fluorescence intensity for $\mathrm{AN}^{+} \mathrm{N}^{+}\left(I_{414}\right)$ to that for $\mathrm{TPE}-2 \mathrm{~N}^{+}\left(I_{474}\right)$ could be employed as a sensing signal for HAase detection in urine. The detection limit was as low as $0.0017 \mathrm{U} \mathrm{mL}^{-1}$ (0.81 $\left.\mathrm{mg} \mathrm{mL}^{-1}\right)$. Further experiments showed that only HAase induced the variations in fluorescence, whereas the addition of other species under the same conditions led to almost no fluorescence variation. These results, in combination with the unique specificity of the enzymatic reaction, indicated that the system had superior selectivity toward HAase over other biologically relevant species. The study also showed good results for detecting HAase in biological fluids such as human urine. ${ }^{29}$

\subsection{Pyrophosphate (PPi)}

Pyrophosphate (PPi) is a type of anion that plays a crucial role in numerous cellular biochemical courses because it is the product of adenosine $5^{\prime}$-triphosphate (ATP) hydrolysis below cellular level and it is also involved in DNA replication when catalyzed by DNA polymerase. ${ }^{30}$ The level of PPi in biofluids is an important biomarker for many disorders, such as calcium pyrophosphate deposition that is detected through knee synovial fluid. ${ }^{31}$ In 2019, a fluorescent bioprobe was developed to selectively and sensitively detect PPi anions (Fig. 8). The study embedded TPE within rigid hyperbranched poly (amido amine) (HPA) to make TPE-HPAs, including P1, P2 and P3. PPi has two functions in the fluorescence turn-on process of TPE-HPAs. One is to connect smaller aggregates of TPE-HPAs by electrostatic attraction; the other is to neutralize the positive charges of TPE-HPAs so as to make the HPA framework less rigid, to support the aggregation of TPE units. P2 showed excellent linearity between fluorescent intensity and PPi concentration in the range of $0-1.0 \mu \mathrm{M}$. The LOD of PPi reached nanomolar level with $6.5 \mathrm{nM}$. The prominent selectivity and sensitivity towards PPi allowed TPE-HPAs to accurately detect the PPi concentration in human urine. ${ }^{32}$ 

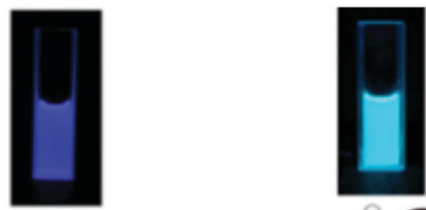

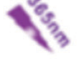

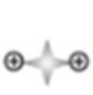<smiles>O=[Hg]=[Os]</smiles>

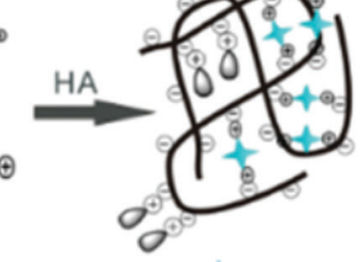

HAase
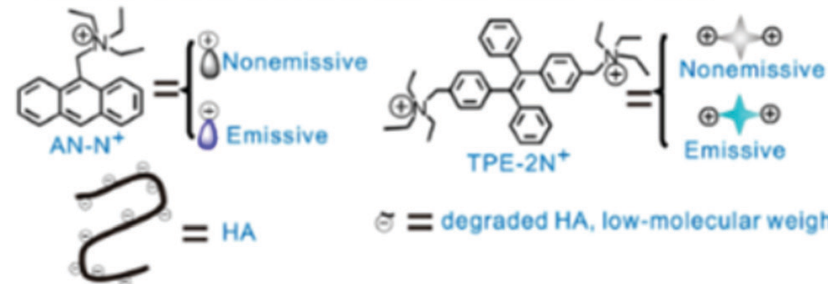

$\odot=$ degraded HA, low-molecular weight fragment
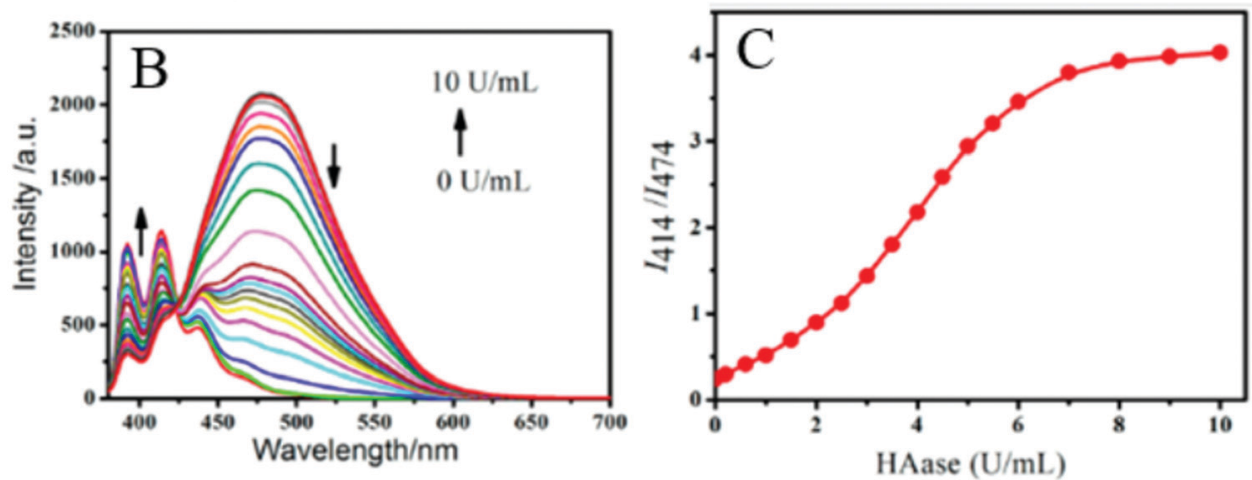

Fig. 7 (A) Schematic illustration of the assay system and its ratiometric fluorescent response to HAase. (B) Fluorescence spectra of the sensing system (4 $\mu \mathrm{M} \mathrm{AN}-\mathrm{N}^{+} / 26 \mu \mathrm{M}$ TPE$-2 \mathrm{~N}^{+} / 0.003 \mathrm{mg} \mathrm{mL}^{-1} \mathrm{HA}$ ) in the absence of HAase and in the presence of different HAase levels in PBS buffer solution ( $0.1 \mathrm{mM}$, $\mathrm{pH}$ 4.3). (C) Fluorescence intensity ratio of $I_{414} / l_{474}$ for the sensing system $\left(4 \mu \mathrm{M} \mathrm{AN}-\mathrm{N}^{+} / 26 \mu \mathrm{M} \mathrm{TPE}-2 \mathrm{~N}^{+} / 0.003 \mathrm{mg} \mathrm{mL}^{-1} \mathrm{HA}\right)$ in PBS buffer solution (0.1 mM, pH 4.3) as a function of HAase level $\left(\lambda_{\mathrm{ex}}=365 \mathrm{~nm}\right)$. Adapted with permission from. ${ }^{29}$ Copyright 2014 American Chemical Society.

In another study, Wang et al. synthesized a Schiff-based AIE probe for detecting PPi. The fluorescence of this probe was weak when dissolved in the solution. However, after $\mathrm{Al}^{3+}$ was added, the probe could provide $\mathrm{N}$ and $\mathrm{O}$ donor centers for bonding with $\mathrm{Al}^{3+}$ to form complexes, in which the rigidity of the probe plane was increased and fluorescence intensity was enhanced as a result. After PPi was added, the probe was disaggregated due to the strong binding ability of PPi to $\mathrm{Al}^{3+}$. Therefore, the fluorescent emission of the complex was reduced. The LOD of PPi was tested to be $0.75 \mu \mathrm{M}$. Moreover, the probe-Al ${ }^{3+}$ complex detected PPi with an "on-off" behavior over other physiologically relevant metal ions, anions and biomolecules, confirming its high selectivity towards PPi. This probe was applied in human urine and achieved good results. ${ }^{33}$

\subsection{Dopamine}

Dopamine is a crucial neurotransmitter of the human nervous system in the brain. It plays an important role in numerous brain functions and behavioral responses. Abnormal amounts of dopamine in the brain can also cause neurological disorders. ${ }^{34}$ Therefore, the Ilanchelian research group developed glutathione-protected silver nanoclusters (GSH-AgNCs) performing as a red emissive nanoprobe (excitation/emission peaks at 490/645 nm) for detecting dopamine. The mechanism was claimed to be the hydrogen bonding interaction between carboxyl groups of GSH-AgNCs and the amino groups of dopamine. The lowest limit of dopamine detection was $0.35 \mathrm{nM}$. This assay was also applied for dopamine quantification in human urine samples. ${ }^{34}$ Recently, Ghosh et al. designed fluorescent gold nanoclusters (AuNCs) by applying trypsin as the ligand. The trypsin-AuNCs emitted red fluorescence that peaked at $655 \mathrm{~nm}$ upon excitation at $520 \mathrm{~nm}$. Such red fluorescence was quenched by the addition of dopamine in the urine sample. This turn-off probe detected dopamine with good selectivity and sensitivity, exhibiting a LOD for dopamine as $0.41 \mathrm{nM}^{35}$ 

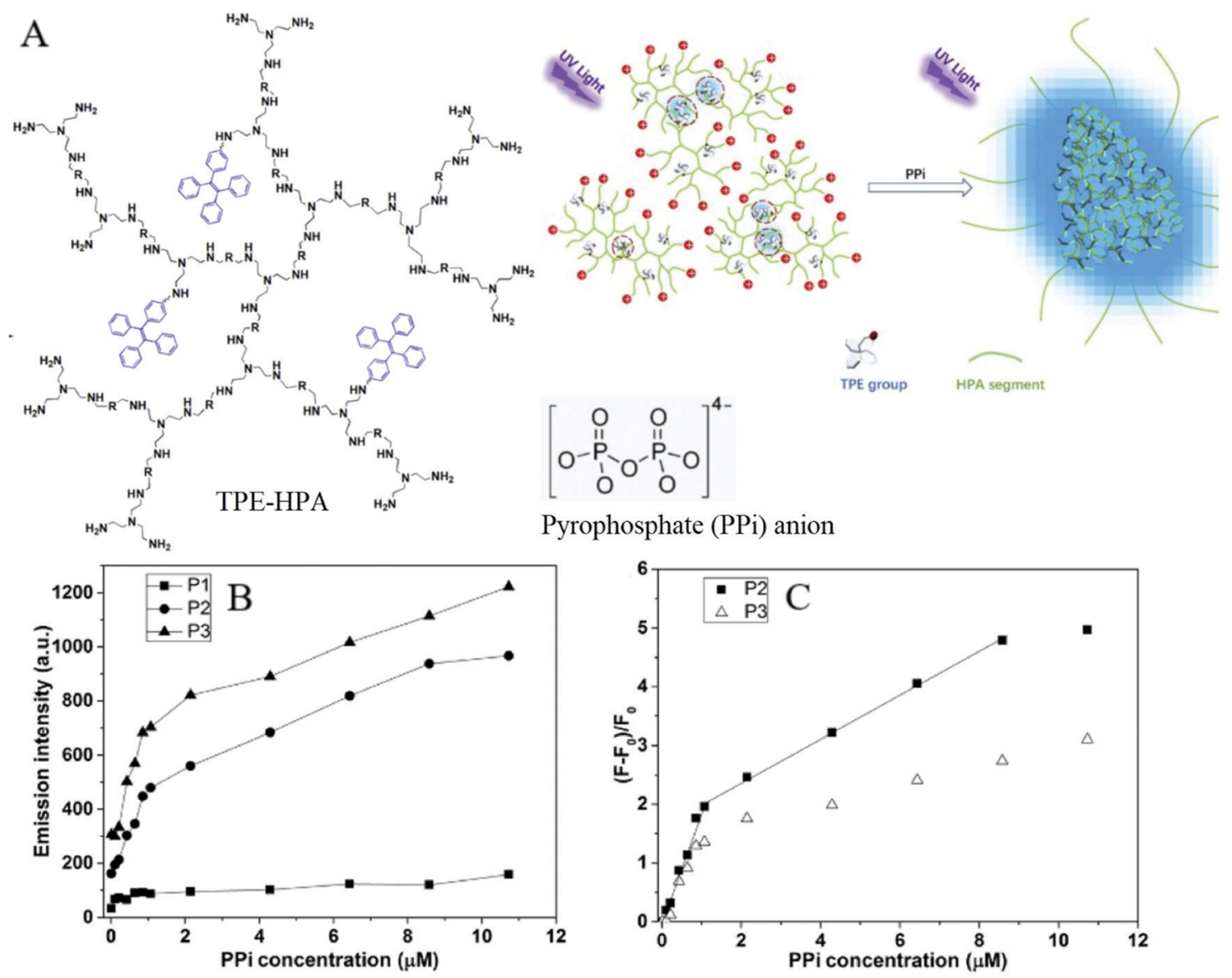

Fig. 8 (A) Chemical structure of TPE-HPAs and schematic illustration of the sensing process of TPE-HPAs. (B) Emission intensity at $475 \mathrm{~nm}$ of different TPE-HPAs versus PPi concentration. (C) Emission change ratio, $\left(F-F_{0}\right) / F_{0}$, versus PPi concentration (TPE $=13 \mu \mathrm{M}, \mathrm{pH}=7.4 ; \lambda_{\mathrm{ex}}=360 \mathrm{~nm}$, slit widths of excitation and emission are 10 and $10 \mathrm{~nm}$, respectively) Adapted with permission from ref. 32. Copyright 2019 Elsevier B. V.

\subsection{Others}

Varying amounts of heavy metal ions are required by living organisms. They are used to maintain various biochemical and physiological functions at low concentrations. They are toxic when they exceed threshold concentrations and can damage to the organism. ${ }^{36,37}$ Mercury ion $\left(\mathrm{Hg}^{2+}\right)$ is one of the most toxic metal ions and can cause severe health problems. $\mathrm{Hg}^{2+}$ can enter the food chain and accumulate in the human body. Moreover, it can result in damage to the central nervous system, brain, and kidneys. ${ }^{38}$ Zhang et al. reported a fluorescent sensor TPE-QN for evaluating $\mathrm{Hg}^{2+}$ levels. This probe was based on TPE functionalized quinolinium salts with hexafluorophosphate $\left(\mathrm{PH}_{6}{ }^{-}\right)$and showed strong red emission. However, in the presence of $\mathrm{I}^{-}$, the emission of TPE-QN was dramatically quenched due to the synergistic electrostatic interactions and dramatic collisions between aggregates of TPE-QN and $\mathrm{I}^{-}$. Since $\mathrm{Hg}^{2+}$ has high affinity to $\mathrm{I}^{-}$, the complex of TPE-QN and $\mathrm{I}^{-}$(TPEQN-I) could be used for the fluorescence turn-on detection of $\mathrm{Hg}^{2+}$. The LOD of $\mathrm{Hg}^{2+}$ was determined to be $71.8 \mathrm{nM}$ and the probe was proved to be usable in real urine samples. ${ }^{38} \mathrm{Zn}^{2+}$ plays an important role in many biological processes, such as cell growth, protein metabolism immunity and neural signal transmission. Abnormal levels of $\mathrm{Zn}^{2+}$ can cause immune deficiency, growth retardation, and serious disease. Free $\mathrm{Zn}^{2+}$ exists in some tissues, including intestines, retina and pancreas. It undergoes a complex process to function as a signaling agent for biological processes. ${ }^{39}$ Wen et al. synthesized an AIE sensor 1-(2-hydro-xynaphthylmethylene)-2-(2hydroxybenzylidene) hydrazine that could detect $\mathrm{Zn}^{2+}$ by selectively turning on the AIE fluorescence response. The sensor could be applied to track $\mathrm{Zn}^{2+}$ in real time and the detection limit was as low as $46 \mathrm{nM}$. The study also showed a simple and effective filter paper method to detect $\mathrm{Zn}^{2+}$ in human urine. ${ }^{39}$

Trypsin is an enzyme that helps to digest protein and is produced by the pancreas. An abnormal trypsin level can cause malabsorption and pancreatitis. It can also serve as a biomarker for many diseases, such as cardiovascular and neurodegenerative diseases. ${ }^{21}$ Zhao et al. designed fluorescent strips of electrospun fibers for detecting trypsin in urine. Their PSMAPhB + TPE/Pro strip could provide ratiometric color change when urine trypsin presented. As shown in Fig. 9, the TPE derivative along with phloxine $\mathrm{B}(\mathrm{PhB})$ was grafted on strip fibers. When protamine was added, the emission of PhB was quenched, while the emission of TPE derivative @ $472 \mathrm{~nm}$ was unchanged. On the other hand, once trypsin existed with the probe, fluorescence of $\mathrm{PhB}$ (a) $574 \mathrm{~nm}$ was recovered, 


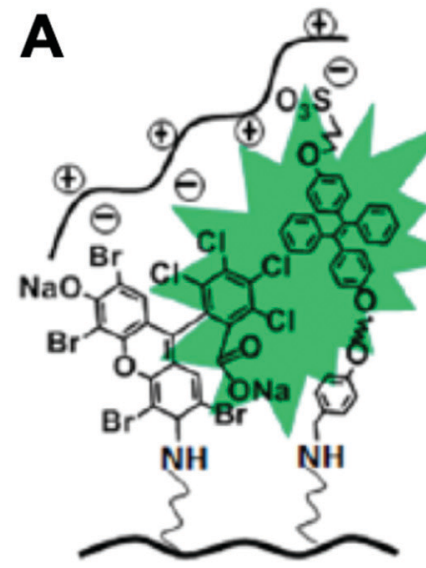

PSMA-PhB+TPE/Pro

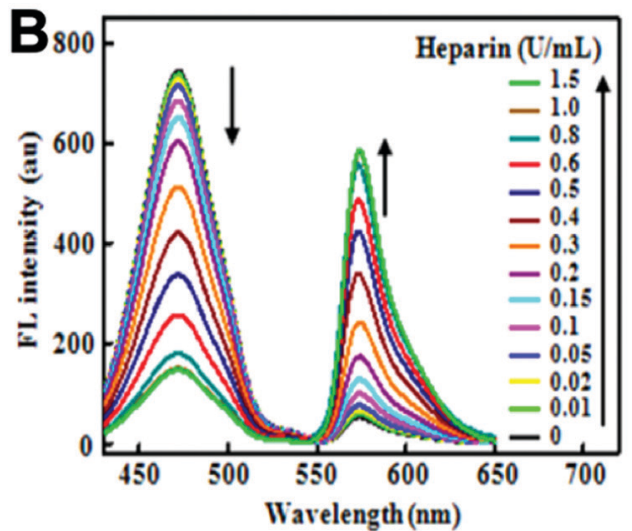

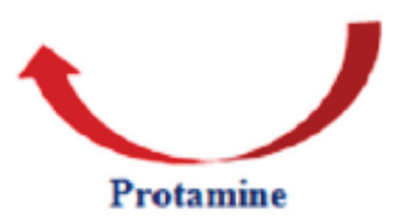

Protamine

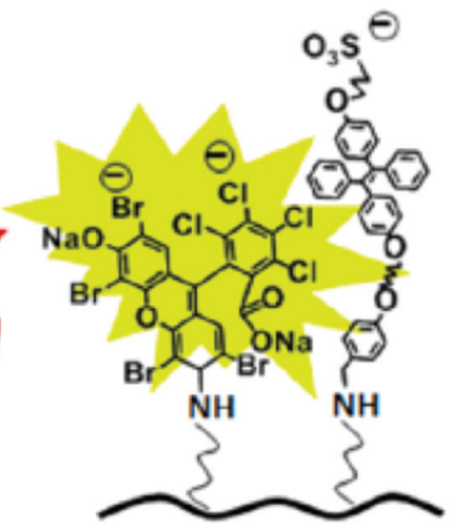

PSMA-PhB+TPE

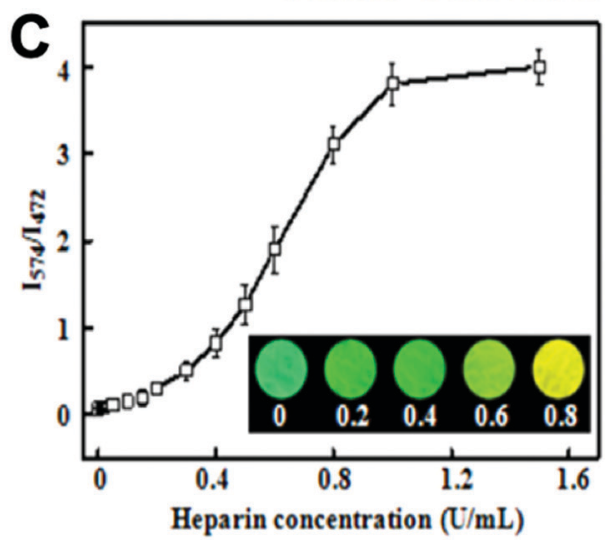

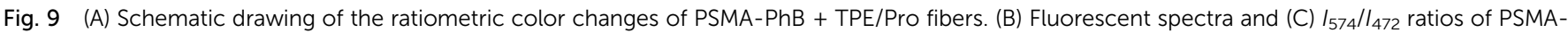

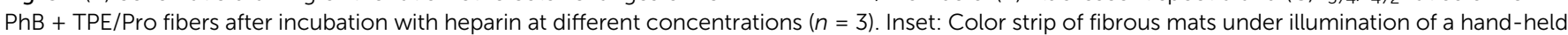
ultraviolet lamp. Adapted with permission from ref. 40. Copyright 2017 American Chemical Society.

accompanying the turn-off of TPE fluorescence. Therefore, the fluorescent intensity ratio of $I_{574} / I_{472}$ could be applied to ratiometric detection of trypsin. The strip turned from cyan to yellow when trypsin level increased to $8 \mu \mathrm{g} \mathrm{mL}{ }^{-1} .40$

D-Glucose is a necessity for living organisms and serves as the energy source for many biological processes. An abnormal glucose level in blood or urine implies a biological dysfunction. ${ }^{41}$ In 2010, Liu et al. developed a TPE-based fluorescent sensor 1 that was functionalized with two boronic acid units (Fig. 10). When the aggregates were suspended in an acidic buffer $(\mathrm{pH}=4.34)$, blue light emitted. The emission intensity began to decrease when the $\mathrm{pH}$ value of the buffer increased above 8.61 and became very weak at $\mathrm{pH}>9.74$. Furthermore, the emission intensity of sensor 1 also enhanced with the increase in the concentration of glucose. When the glucose level increased to $5 \mathrm{mM}$, the solution of sensor 1 became very emissive. However, further increase in the glucose concentration decreased the fluorescence intensity of sensor $1 .^{41}$

Bacterial lipopolysaccharide (LPS) is a biomarker for monitoring bacterial urinary tract infections (UTIs) which occur frequently in all age groups especially in females. LPS serves as a marker of UTIs independent of antibiotic therapy, due to the LPS released by dead bacteria. ${ }^{42}$ Jiang et al. designed an AIE probe, TPEPyE, with positive charges which could bind to negatively charged LPS through electrostatic interactions (Fig. 11). Their results showed over 22-fold fluorescence enhancement when LPS presented in the samples. The plot at $515 \mathrm{~nm}$ versus the LPS concentration exhibited a good linear relationship, with an LPS concentration range up to $0.5 \mu \mathrm{M}$ $\left(5 \mu \mathrm{g} \mathrm{mL}^{-1}\right)$ and a LOD as low as $370 \mathrm{pM}\left(3.7 \mathrm{ng} \mathrm{mL}^{-1}\right)$. TPEPyE also showed good sensitivity and selectivity in artificial urine. Moreover, this probe could distinguish Gram-negative and Gram-positive bacteria, making it a promising sensor for clinical detection of urinary tract infections. ${ }^{42}$

Adenosine $5^{\prime}$-triphosphate (ATP) is a primary energy source in living organisms and also has extracellular functions such as mediating vasodilation and pain perception. Additionally, ATP also affects local tissue changes in neurotransmission and neuromodulation. ${ }^{43}$ As shown in Fig. 12, Liu et al. designed a probe based on glutathione modified silver nanoclusters (AgNCs/GSH). Zinc ion enhanced the emission of AgNCs/GSH because it coordinated with the probe to result in the aggregation of AgNCs/GSH. When ATP was present, $\mathrm{Zn}^{2+}$ preferred to bind with the ATP via strong $\mathrm{Zn}-\mathrm{O}-\mathrm{P}$ bonds and the binding between $\mathrm{Zn}^{2+}$ and AgNCs/GSH was dissociated as a result. Therefore, the fluorescent intensity of $\mathrm{AgNCs} / \mathrm{GSH}$ would decrease by increasing the ATP concentration. The ATP could 
A
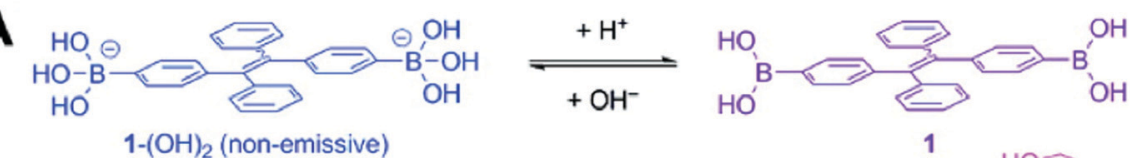

\begin{tabular}{l||l}
$-\mathrm{Glu}$ & $+\mathrm{H}_{2} \mathrm{O}$
\end{tabular}$-\mathrm{H}_{2} \mathrm{O}$
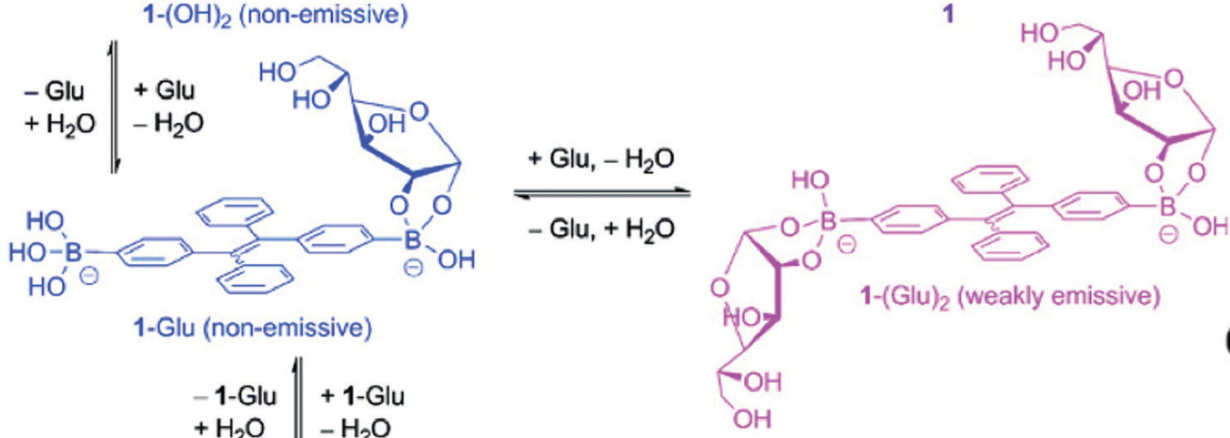

1
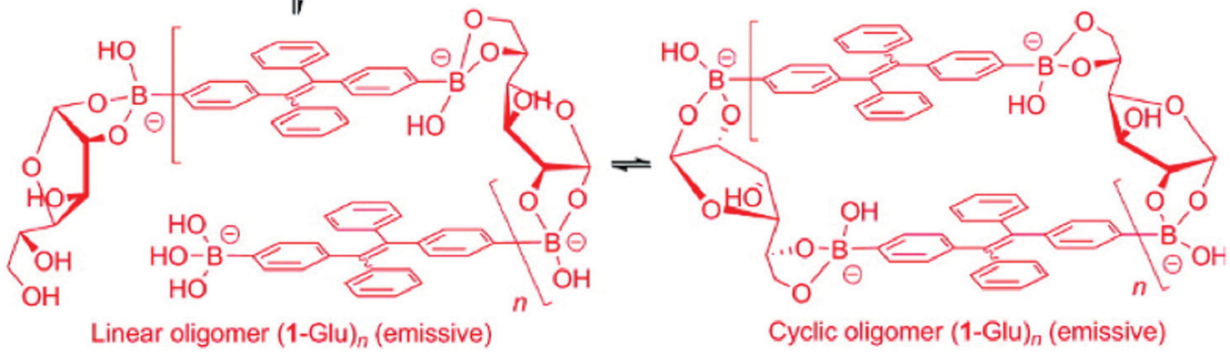

Cyclic oligomer (1-Glu) $)_{n}$ (emissive)

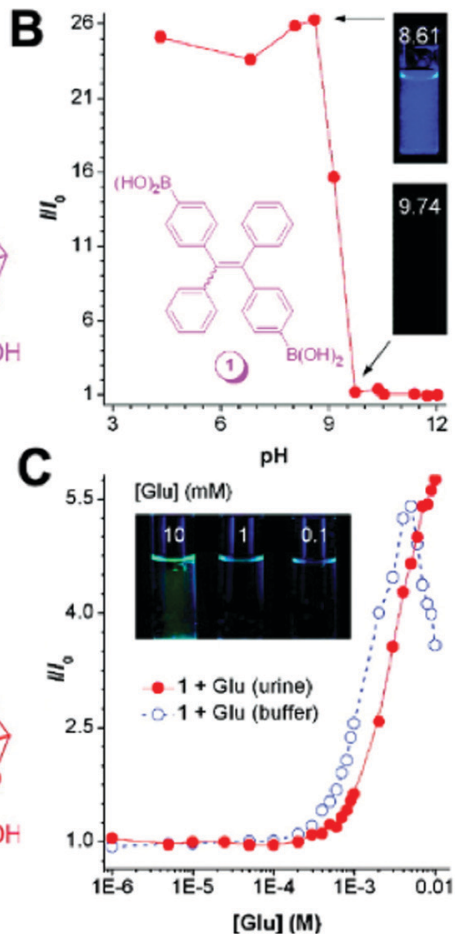

Fig. 10 (A) Proposed mechanism for the process of glucose-specific sensing by AlE-active sensor 1 . (B) Change in the FL intensity (I//0) of sensor 1 at $440 \mathrm{~nm}$ with pH values; $I_{0}$ is the $\mathrm{FL}$ intensity at $\mathrm{pH}$ 12.01. Inset: Photographs of the mixtures of 1 in the aqueous buffers at $\mathrm{pH} 8.6$ and 9.7 taken under the illumination of a hand-held UV lamp $\left(\lambda_{\mathrm{ex}}=365 \mathrm{~nm}\right)$. (C) Changes in the FL intensity $\left(/ / /_{0}\right)$ of $1(50 \mu \mathrm{M})$ with glucose concentration in artificial urine (red circle) and carbonate buffer (blue circle). $I_{0}$ is the FL intensity in the absence of glucose. The inset in panel $C$ shows photographs of mixtures of 1 ( $50 \mu M$ ) in artificial urines containing different amounts of glucose taken under UV illumination. Adapted with permission from ref. 41 . Copyright 2010 American Chemical Society

be monitored within the linear range of $1-110 \mu \mathrm{M}$ and the LOD was $0.8 \mu \mathrm{M}$. The method has been successfully applied in human urine samples. ${ }^{44}$

Adenosine is an endogenous nucleoside in biochemical processes and is also a neuromodulator for promoting sleep and suppressing arousal. It regulates the blood flow to organs through vasodilation. Scientists have found the concentration of adenosine in the extracellular tissue surrounding tumors to be higher than under healthy conditions. Hence, adenosine is a possible biomarker for cancer and other diseases. ${ }^{45} \mathrm{Hu}$ et al. designed a fluorescent probe TPE-ABA through combining an AIE molecule TPE- $2 \mathrm{~N}^{+}$and an adenosine-specific aptamer (ABA) for detecting adenosine in human urine. The aptamer can bind to the adenosine and form a G-quadruplex that restricts the free rotation of phenyl rings in TPE- $2 \mathrm{~N}^{+}$. The linear range of this detection assay is $10 \mathrm{pM}$ to $0.5 \mu \mathrm{M}$ with a $R^{2}$ of 0.996 and the LOD of 10 pM. $^{45}$

Spermine is a natural polyamine found in body fluids. It plays an important role in cell growth and proliferation. The detection of spermine in urine may indicate malignant tumors. Hence, such detection may help evaluate the effectiveness of cancer treatment and relapse. ${ }^{46}$ As show in Fig. 13, a fluorescent probe 1@CB[7] demonstrated high selectivity and sensitivity towards spermine within the linear range $0-12 \mu \mathrm{M}$ and an LOD of $1.0 \mu \mathrm{M}$. The probe is a supramolecular system constructed between an AIE molecule and cucurbit[7]uril (CB[7]).
Most importantly, the detection range covers the requirement for early diagnosis of cancer, which is $1-10 \mu \mathrm{M}$ in urinary spermine. ${ }^{46}$ Therefore, 1@CB[7] holds promise for development into a fluorescent sensor for clinical application.

Glutathione (GSH, L- $\gamma$-glutamyl-L-cysteinyl-glycine) is an essential endogenous antioxidant that serves many biological functions. The concentration of GSH ranges at micromolar levels in body fluids including urine and saliva. Abnormal levels of GSH may imply many disorders, such as cancer, liver damage, and hearing problems. Sensitive methods are desirable for better understanding of the mechanisms of such diseases. ${ }^{47}$ In 2017, Zhang et al. designed an anionic TPE derivative TPE3 that was used as an AIE probe for detection of GSH. First, negatively charged $\mathrm{MnO}_{2}$ was employed as both a positive charge protector for $\mathrm{SiO}_{2} \mathrm{NPS}$ and a recognition unit for GSH. When GSH presented, $\mathrm{Mn}^{2+}$ was released and the positive charges of $\mathrm{SiO}_{2} \mathrm{NPs}$ were exposed for the binding and aggregation of TPE3, inducing strong fluorescence emission. A linear range from 0.5 to $100 \mu \mathrm{M}$ was reported and the LOD was $200 \mathrm{nM}$. The study also demonstrated that probing TPE3 is feasible in testing biological samples. ${ }^{47}$

Certain drugs, such as chlortetracycline (CTC), carbidopa, and erythromycin (ERY), can also be regarded as biomarkers for urine drug testing. CTC is a broad-spectrum antibiotic that has antimicrobial activity against Gram-positive and Gram-negative bacteria. It is used as a drug for infectious diseases and as a 
A
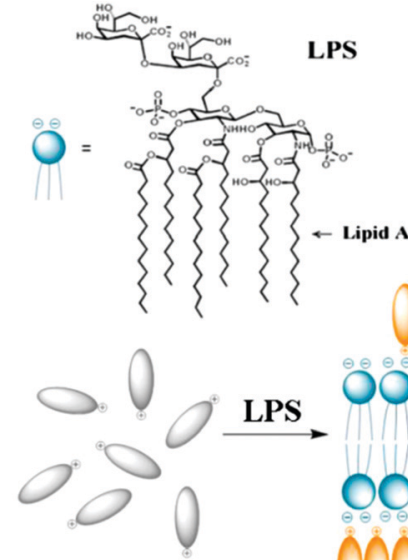

in artificial urine sample weakly emissive
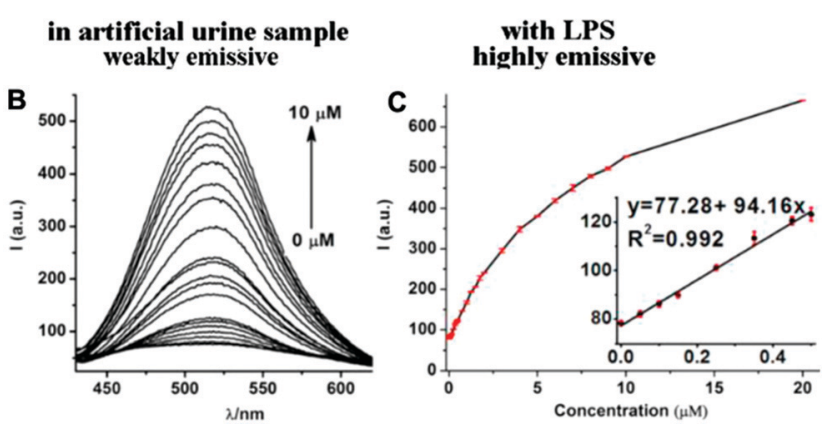

Fig. 11 (A) Structure of TPEPyE and schematic illustration of LPS sensing process. (B) Fluorescence intensity of TPEPyE $(4 \mu \mathrm{M}$ in artificial urine samples) with different concentrations of LPS $(0-10 \mu \mathrm{M}), \lambda_{\mathrm{ex}}=405 \mathrm{~nm}$. (C) The plot and linear fitting of fluorescence intensity at $519 \mathrm{~nm}$ vs. the concentration of LPS $(0-10 \mu \mathrm{M})$. Insets present the plot and linear fitting of fluorescence intensity at $519 \mathrm{~nm}$ vs. the concentration of LPS $(0-0.5 \mu \mathrm{M})$. Adapted with permission from ref. 42. Copyright 2016 Elsevier B.V.

feed additive for animal growth. However, extensive use of CTC may result in antibiotic residues in food and lead to serious side effects for some hypersusceptible individuals. ${ }^{48}$ Recently, a zinc-based metal-organic framework of pyromellitic acid (Zn-BTEC) has been developed to detect CTC by applying its intrinsic AIE property. The sensing mechanism is based on the porous structure of Zn-BTEC whereby the CTC can defuse into the rigid metal-organic structure then aggregate to enhance fluorescence. This bioprobe can also discriminate CTC from other tetracycline antibiotics. Testing has shown good sensitivity and selectivity of CTC in real urine with the LOD of $28 \mathrm{nM}^{48}$

Carbidopa used as a drug helps to increase the delivery levels of dopamine to the central nervous system by inhibiting the decarboxylation and inactivation of L-DOPA in peripheral tissues. $^{35}$ Ghosh et al. designed a fluorescent nanoprobe trypsin-AuNCs that could detect carbidopa under the excitation wavelength of $520 \mathrm{~nm}$ via a fluorescence quenching process. The LOD for carbidopa was tested to be $6.5 \mathrm{nM}$ and the probe worked successfully in human urine. ${ }^{35}$ ERY has been used since the 1950 s and is the most prevalent antibiotic for the treatment of infections. It is usually applied in the treatment of upper respiratory and soft tissue infections caused by microorganisms. However, the broad use of ERY has resulted in overdoses which can lead to serious effects including diarrhea, hearing loss, and liver failure. Therefore, it is essential to detect ERY for the protection of public health. ${ }^{49}$ Zhang et al. developed a fluorescent probe for determination of the macrolide antibiotic ERY. Fluorescent gold nanoclusters (AuNCs) were coated with silica first to

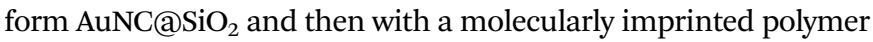
(MIP) to produce AuNCs@SiO ${ }_{2}$-MIPs. This probe had strong fluorescent emission that decreased with enhancement of ERY concentration in the range of 0.1 to $11.9 \mu \mathrm{M}$. Furthermore, this probe was tested in spiked human urine and saliva (Table 3). ${ }^{49}$

Urinary testing offers an inexpensive, simple, and rapid way to test people suffering from renal disease or from asymptomatic beginnings of disease such as liver disease and diabetes. ${ }^{13}$ However, most of these tests must be undertaken in the clinical laboratory. The AIE bioprobe as a novel fluorescent material can be used qualitatively and quantitatively to detect biomarkers of disease. If the assay is tolerant of real human specimens, it is promising for application in the reallife environment. Design and fabrication of simple biomarker assessment kits for clinical diagnostics or home use screening are ongoing in several laboratories. Here we report three feasible strip designs for biomarker detection through fiber or paper-based sensors. Zhao et al. designed an electrospun fibrous strip that could exhibit a straightforward result of ratiometric color changes through naked eye observation when urine trypsin presented. ${ }^{40} \mathrm{Yu}$ et al. developed a paper-based sensor for visual detection of CTC. The strip was made of a polyvinylidene fluoride microporous filter membrane. After CTC presented, the strip showed a bright green color under $365 \mathrm{~nm}$ UV illumination. ${ }^{48}$ Another filter paper-based sensor for visual detection of $\mathrm{Zn}^{2+}$ was also developed. This method was based on colorimetric and selective AIE fluorescence responses in the solid state to detect $\mathrm{Zn}^{2+}$ by the naked eye. The fluorescence intensity was gradually increased in the filter paper by increasing the $\mathrm{Zn}^{2+}$ concentration and the visual LOD of $\mathrm{Zn}^{2+}$ was reduced to $20 \mu \mathrm{M} .{ }^{39}$

\section{Saliva}

Saliva is a clear and mild acidic (pH 6-7) mucoserous exocrine secretion. It is created from the salivary glands and from gingival crevicular fluid. The average daily flow amount of saliva is between 1 and $1.5 \mathrm{~L}$. Saliva is a dilute fluid of which more than $99 \%$ is water. The constituents of saliva include a variety of electrolytes: sodium, potassium, calcium, magnesium, bicarbonate, and phosphates. There are also immunoglobulins, proteins, enzymes, mucins, and nitrogenous products such as urea and ammonia. ${ }^{4}$ The functions of saliva can be categorized into five aspects for maintaining oral health and ecological balance: lubrication, clearance, tooth integrity, antibacterial activity, and taste. ${ }^{4}$ Saliva is a valuable body fluid for oral health. Various research into saliva dysfunction relates to diseases or certain medications. ${ }^{4}$ Since saliva is easily collected and is noninvasive source of information, it becomes a useful body fluid for medical diagnosis. 

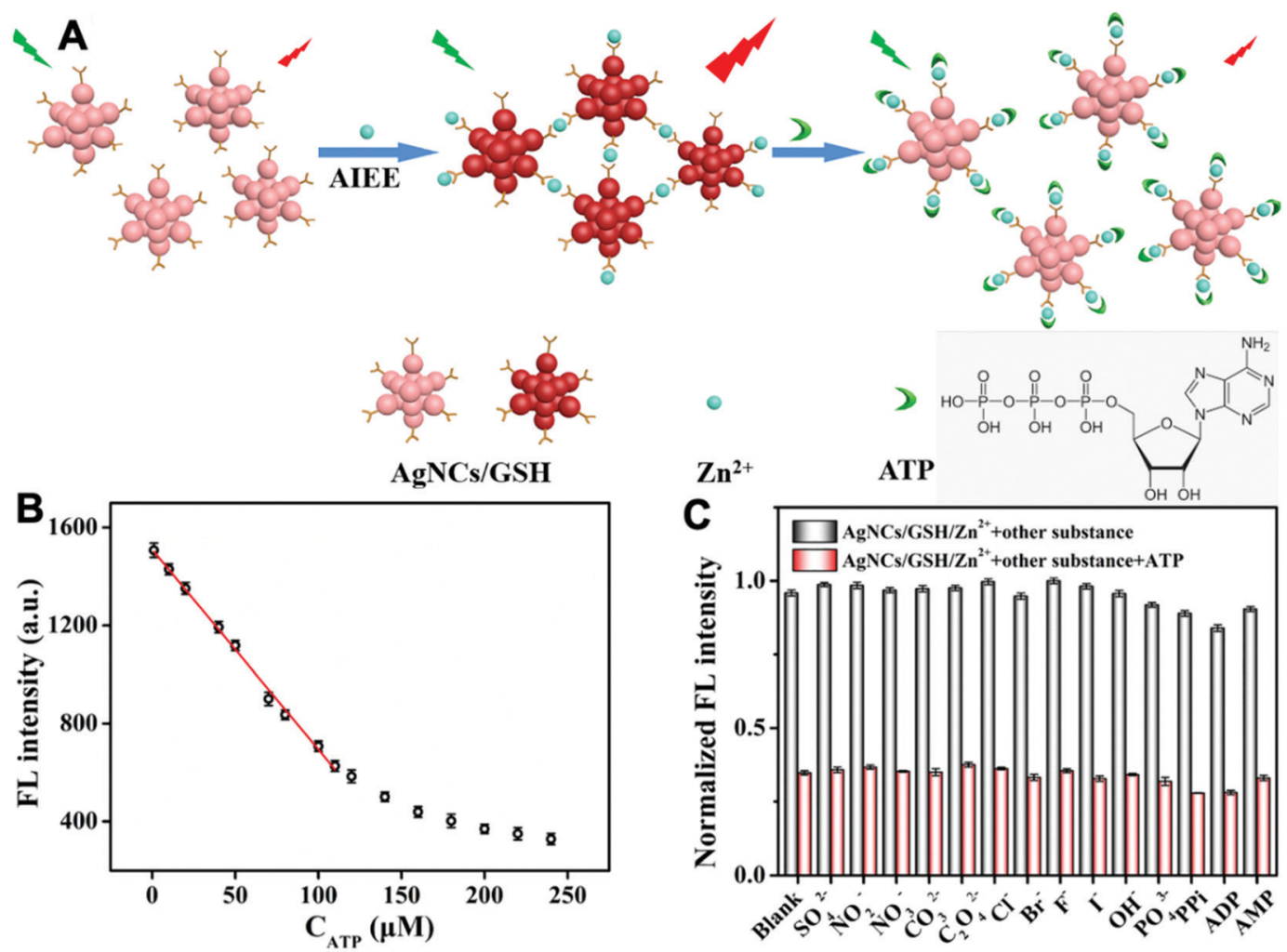

Fig. 12 (A) Detection strategy for ATP via inhibiting the AIE enhancement of AgNCs/GSH triggered by zinc ions. (B) The working curve between the fluorescence intensities and the concentration of ATP. (C) Selectivity experiments for ATP detection. The concentration of $Z n^{2+}$ and anions is $240 \mu M$. The concentration of ATP and analogs is $100 \mu \mathrm{M}$. Adapted with permission from ref. 44. Copyright 2019 Elsevier B. V.

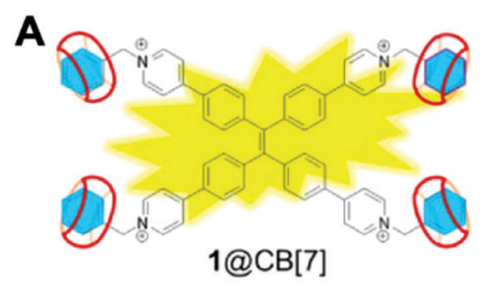

Fluorescence "ON"

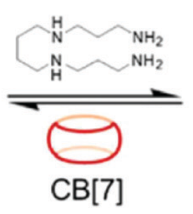

$\mathrm{CB}[7]$

Fig. 13 (A) Proposed mechanism of fluorescent probes 1(aCB[7] for spermine detection. (B) The fluorescence intensity of 1@CB[7] at 537 nm as a function of the concentration of spermine $(0-40 \mu \mathrm{M})$. Inset shows the plot and linear fitting of fluorescence intensity at $537 \mathrm{~nm} v \mathrm{vs}$. the concentration of spermine $(0-12 \mu \mathrm{M})$. Adapted with permission from ref. 46. Copyright 2018 Elsevier B.V.

Salivary samples can be applied to reflect the status of body health. It is usually used in the diagnosis of dental disorders and is used for caries risk analysis, periodontal disease genotypes, and biomarker identification of periodontal disease. Other dental diseases that can be detected include salivary gland disease and dysfunction and candida infections. Currently, saliva is also used for the detection of viral diseases, gastric ulcers and cancers, liver disease, and tuberculosis. ${ }^{4}$ Saliva can be used to monitor levels of polypeptides, steroids, antibodies, alcohol, and various drugs. Moreover, some research has been conducted to determine the clinical value of saliva for detection of cancer and preterm labor. Growth factors can also be found in saliva and they play an important role in wound healing and oral health maintenance. ${ }^{4}$ The commercial market provides saliva test strips that can be used at home to detect alcohol, drugs, and nitric oxide. Most of the methods collect saliva through an absorbent pad, apply a chemical solution, and detect color change to indicate the result. Some methods commonly use point-of-care (POC) devices to screen for diseases such as HIV. ${ }^{4}$ There still are many opportunities to develop optical sensors for biomolecules in saliva such as dental disease detection and drug abuse area. However, commercial devices based on analytes detection in saliva are rare and nonpopular.

Here we introduce a few AIEgens used in salivary biomarker detection (Table 4). The first salivary biomarker is HSA. 
Table 3 AlEgens for urine biomarker detection
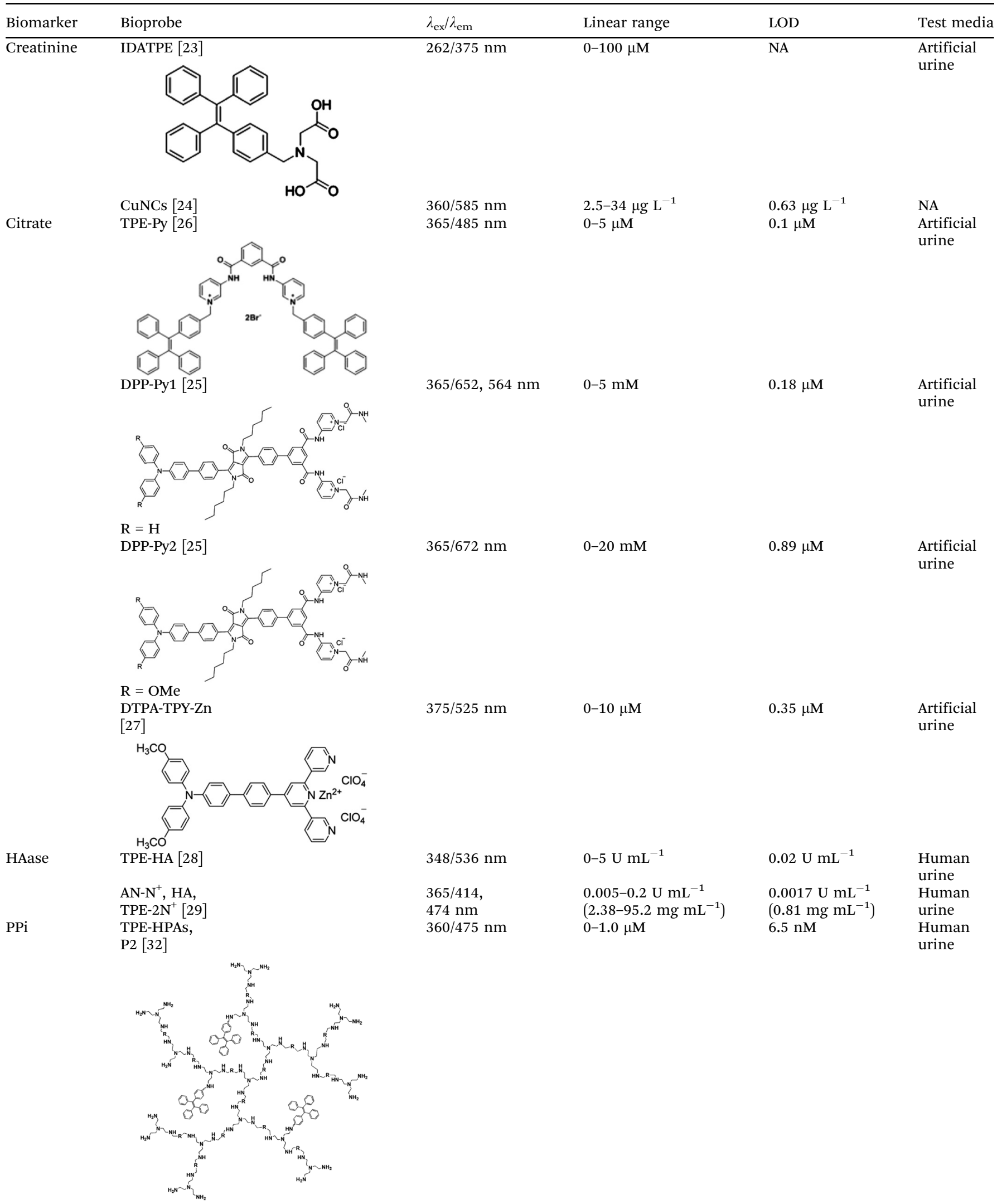
Table 3 (continued)

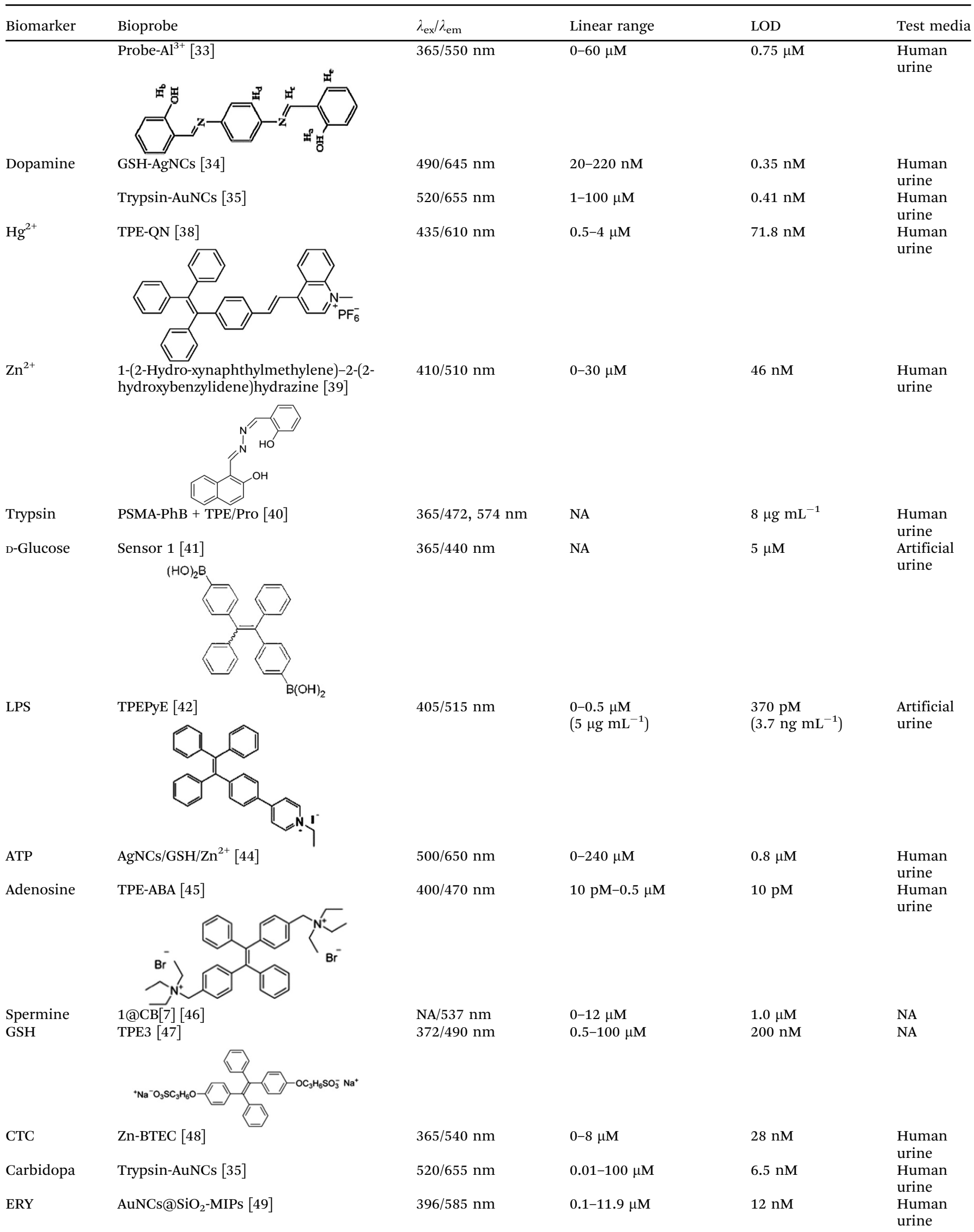


Table 4 AlEgen for saliva biomarker detection

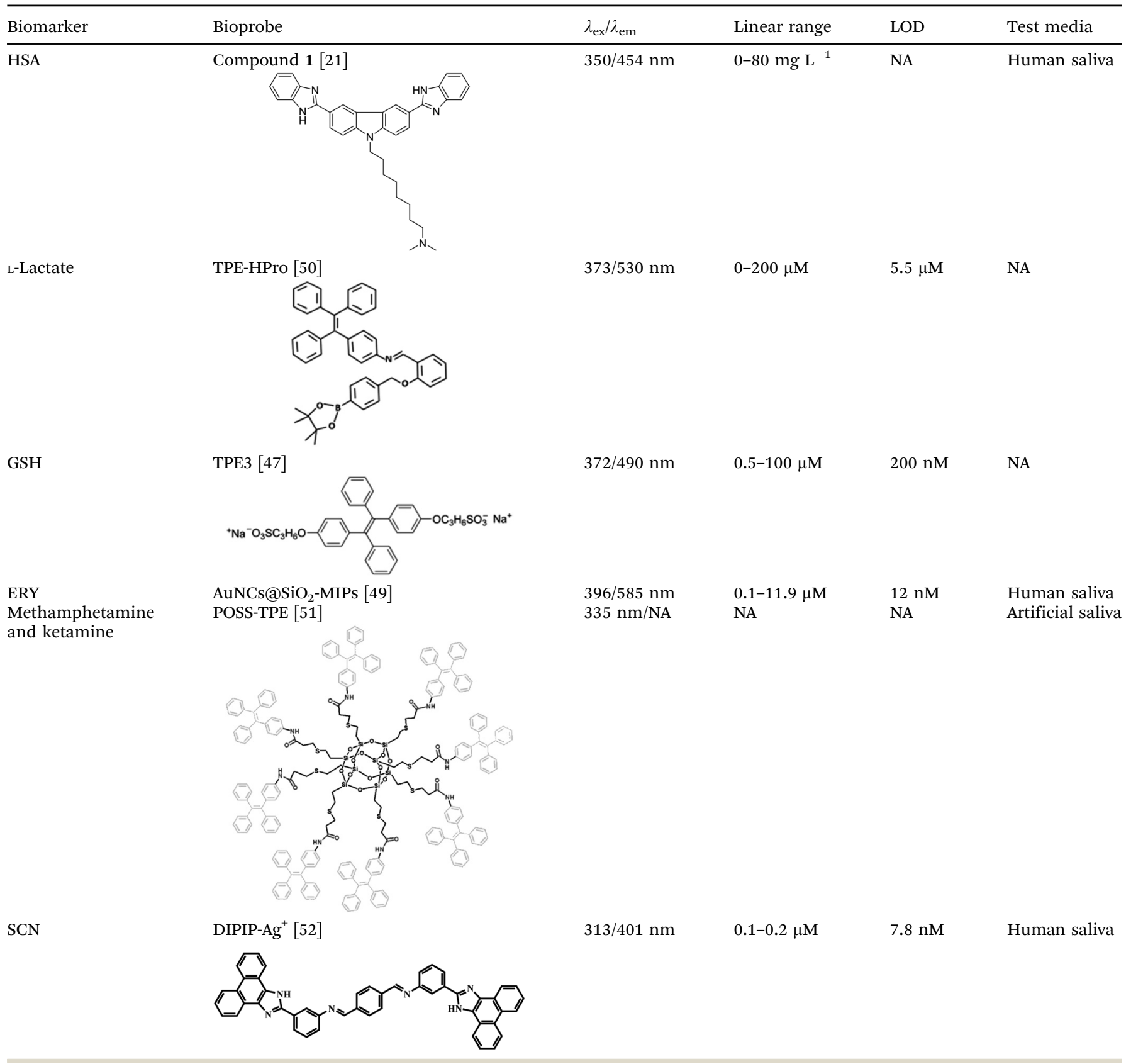

According to references, the normal concentration of HSA in saliva is no more than $0.5 \mathrm{~g} \mathrm{~L}^{-1}$. Beyond this range, various health issues may be associated with abnormal levels, such as type-2 diabetes or stomatitis. ${ }^{21}$ As shown in Fig. 14, the bioprobe compound $\mathbf{1}$ already mentioned in the urine section can also evaluate albumin in saliva. In this assay, the human saliva samples must be diluted 100 -fold, which means that only $0.2 \mu \mathrm{L}$ of saliva is sufficient for albumin quantification.

L-Lactate is an important metabolite for human health and is produced by anaerobic respiration of glucose. It is a biomarker for physiological fatigue and disorders including hyperlactatemia, cardiogenic shock, respiratory disease, tissue hypoxia, and liver disease. It can also express the extent of physical activity, serving for analyzing physiological fatigue of athletes, soldiers, and manual laborers. ${ }^{50}$ In 2017 , Zhang et al. developed a new AIE-active fluorophore TPE-HPro that can combine with L-Lactate oxidase to determine L-Lactate in aqueous fluid. The assay exhibited a LOD of $5.5 \mu \mathrm{M}$ and a linear correlation in the concentration range of $0-200 \mu \mathrm{M}$. Selectivity testing showed that this probe could be used in a protein-rich environment. Zhang and colleagues also identified the optimal conditions for this test including $\mathrm{pH}$, concentration of TPE-HPro, and L-Lactate oxidase activity. They concluded that this bioprobe had high potential for L-Lactate detection in sweat and saliva. ${ }^{50}$

As noted, GSH plays an important role in biological functions and can also be measured in saliva. Absence of GSH may 

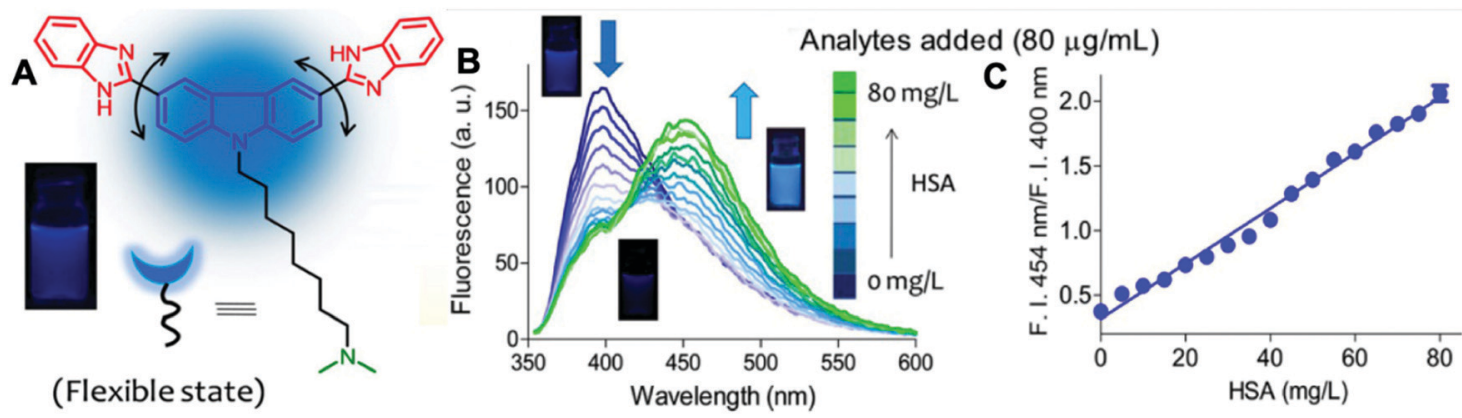

Fig. 14 (A) Chemical structure of compound 1. (B) Fluorescence titration of compound $1\left(\lambda_{\mathrm{ex}}=350 \mathrm{~nm}, 10 \mu \mathrm{M}\right)$ with HSA $\left(0-80 \mathrm{mg} \mathrm{L}{ }^{-1}\right)$ at pH 7.4 in PBS buffer. (C) Ratiometric changes in the emission intensity of compound 1 ( $\lambda_{\text {ex }}=350 \mathrm{~nm}, 10 \mu \mathrm{M}$ ) upon the addition of HSA at pH 7.4 in PBS buffer. Adapted with permission from ref. 21. Copyright 2018 Wiley-VCH Verlag GmbH \& Co. KGaA, Weinheim.

indicate the presence of cancer. Normally, it presents micromolar concentration in saliva. ${ }^{47}$ The AIEgen TPE3, combined with $\mathrm{SiO}_{2} \mathrm{NPs}$ to form AIE-SiO ${ }_{2} \mathrm{NPs}$, can detect GSH through fluorescence enhancement. The linear range of GSH detection is from 0.5 to $100 \mu \mathrm{M}$ and the LOD is $200 \mathrm{nM} .{ }^{47}$ Previous study had tested this probe in urine sample but not in saliva. However, the research of Zhang et al. ${ }^{49}$ enabled GSH detection through saliva in the next stage. For salivary ERY detection, as explained in the urine section, probe AuNCs@SiO ${ }_{2}$-MIPs can be used for detecting ERY through the fluorescence turn-off process. This assay has also been tested in human saliva. ${ }^{49}$ Moreover, illicit synthetic drugs including methamphetamine and ketamine have been prevalent in recent years. The abuse of synthetic drugs in situations such as drugged driving can become severe social problems and threats to public health. Therefore, fast and effective detection of illicit drugs is in highly demand. ${ }^{51}$ Recently, a fluorescence chemosensor with the AIE property was designed for detecting synthetic drugs including methamphetamine and ketamine. The probe was synthesized with an eight TPE-modified polyhedral oligomeric silsesquioxane (POSS) dendrimer and exhibited strong emission due to noncovalent interaction forces. The probe POSS-TPE showed highly sensitive and selective detection of methamphetamine and ketamine in artificial saliva. ${ }^{51}$

$\mathrm{Bu}$ et al. developed a probe based on a phenanthro[9,10- $d]$ imidazole derivative (DIPIP) and $\mathrm{Ag}^{+}$for detecting $\mathrm{SCN}^{-}$in smoker saliva samples. The concentration of $\mathrm{SCN}^{-}$in the saliva samples of tobacco smokers is usually higher than that of nonsmokers. The LOD for $\mathrm{SCN}^{-}$is as low as $7.8 \mathrm{nM}$ by employing DIPIP-Ag ${ }^{+}{ }^{52}$ At the time of writing, there are no AIEgen based paper strips or other applications for analyte diagnosis in saliva samples. Recently, however, a few AIEgen researchers have tried to explore their application in dental disease through monitoring enzymes or bacteria in saliva samples. The development of paper strip applications for saliva testing is anticipated.

\section{Sweat}

Sweat is produced by eccrine and apocrine glands which are in the dermal level of skin and terminate in secretory canals.
The sweat can flow into the skin surface and hair follicles. ${ }^{53}$ Most of the sweat consists of water and electrolytes. There are also amounts of minerals, lactic acid, and urea in sweat. The mineral content includes sodium, potassium, calcium, and magnesium. ${ }^{5}$ Sodium and chloride are the primary electrolytes in sweat. Many other elements are also present in sweat, such as zinc, copper, iron, chromium, nickel, and lead. ${ }^{6,7}$ In addition, approximately $1 \%$ of the sweat consists of proteins such as immunoglobulins and glycoproteins. Sweat is at moderate acidic to neutral $\mathrm{pH}$ levels, normally between 4.5 and 7.0. ${ }^{54}$ Currently, applications of sweat are as a biofluid for clinical use are limited. There are a few reports of sweat analysis for detection of ethanol, drugs, ions, or metals. ${ }^{55}$ Sweat diagnostics in clinical testing include only one commercial application, which is for infant cystic fibrosis. This test detects chloride concentrations in the sweat. Few researches develop optical sensors for sweat biomolecules detection due to many challenges, such as difficult obtaining sweat samples and no clinical guidelines for sweat analysis. The opportunities of combining optical sensor and wearable device may encourage scientists to study more on this biofluid. There are a few reports of AIEgen which could detect analytes in sweat.

Besides salivary samples, L-Lactate can also be tested in the sweat. The probe TPE-HPro reported by Zhang et al. can determine $\mathrm{s}$-Lactate not only in saliva but also in sweat. This probe works well in a protein-rich specimen. The fluorescent signal can be stable for around 60 minutes. However, further work using this probe to detect $\mathrm{L}$-Lactate in real environments is still needed. For more details about this work, please refer to the saliva section. ${ }^{50}$ Until now, no studies have shown any application based on AIE probes detecting analytes in human sweat. However, scientists are highly interested in sweat detection through wearable devices. Sweat can be sampled easily and is currently assessed through noninvasive methods such as electronic tattoos, bands, or patches. ${ }^{56}$ It can be employed as a diagnostic fluid but there are still many challenges, such as the small sample volume and the potential for contamination from the skin surface. AIEgen can be a valuable bioprobe to develop wearable devices for biomarker detection in human sweat, due to its efficient and fast response to analytes. 


\section{Future opportunities and challenges}

Body fluids are recognized as highly important in medical diagnostics due to their rich information about biomarkers. A wide range of health conditions are revealed by abnormal levels of biomolecules in body fluids. Currently, such biofluids are assessed in pathological laboratories which may not have the capacity of conducting timely analyses, especially in emergency conditions. As we have discussed, fluid analysis can provide easy, rapid, and noninvasive sample collection, at low cost and with highly reliable diagnostics. To realize these merits, a point-of-care (POC) device that includes a bioprobe and analytical device is indispensable. Zhang et al. reported enzymatic biosensor design procedures that include four steps (Fig. 15). This approach adapts engineering science to develop bioprobes in specific areas. It may provide a systematic design procedure for bioprobe products. ${ }^{57}$

Many studies of synthesizing bioprobes with AIE properties exist and these AIEgen probes have demonstrated excellent sensitivity and selectivity in biomarker detection. However, only a few of these bioprobes have been evaluated in real body fluid. Although these probes have presented robust properties in artificial biofluids, interference in clinical scenarios still challenges their performance. Researchers need to test probes through clinical hospital trials in order to understand their practical performance, which will enhance the feasibility of developing AIEgen applications. Besides the design and synthesis of AIEgens, their corresponding POC devices are also in high demand. This means that chemical researchers need to cooperate with biomedical engineers, mechanical engineers, and clinical staff to developing valuable products for patients.

There are a few feasible tests using paper-based strips for analyte detection. There is also a large potential to develop fluorescent strips and corresponding analytical devices. This review reports some AIEgen paper-based detection that has shown good results but still needs further improvement. Shaymaa et al. have developed a smartphone based POC urinalysis device that is based on AIEgen detection under the solution state. There is still the need, however, to design a detection device based on a strip test, due to hygienic requirements and convenience. ${ }^{58}$ The development of AIEgen paper strips and detection devices deserves more attention from researchers. In future, the POC device may be combined with a smartphone application for image analysis. All images and analyzed data will be uploaded to the Cloud for telemedicine; then patient and doctor can diagnose and supervise the analyte level conveniently. Not only urine analysis, also saliva and sweat collected non-invasively need more attention for the development of AIE bioprobes and their related innovative detection methods.

\section{Conclusion}

The bioprobe industry is anticipated to experience rapid growth due to the increasing awareness of early detection and fast screening of diseases. In this review, we have summarized recent progress in applying AIEgens for the detection of biomarkers in body fluids such as urine, saliva, and sweat. It can be concluded that scientists not only need to accelerate AIEgen sensor development for body fluids but also to focus on the performance of AIEgens applied in real environments. The development of a POC device or other wearable device matched with AIE bioprobes also needs further involvement. The direct results obtainable from using simple sensing processes in easily controlled settings for some AIE bioprobes, provides good potential convenient and low-cost packaging of this approach, making use of common technologies such as smartphone data capture and analysis, and data cloud storage. High selectivity and sensitivity of AIE bioprobes integrated with

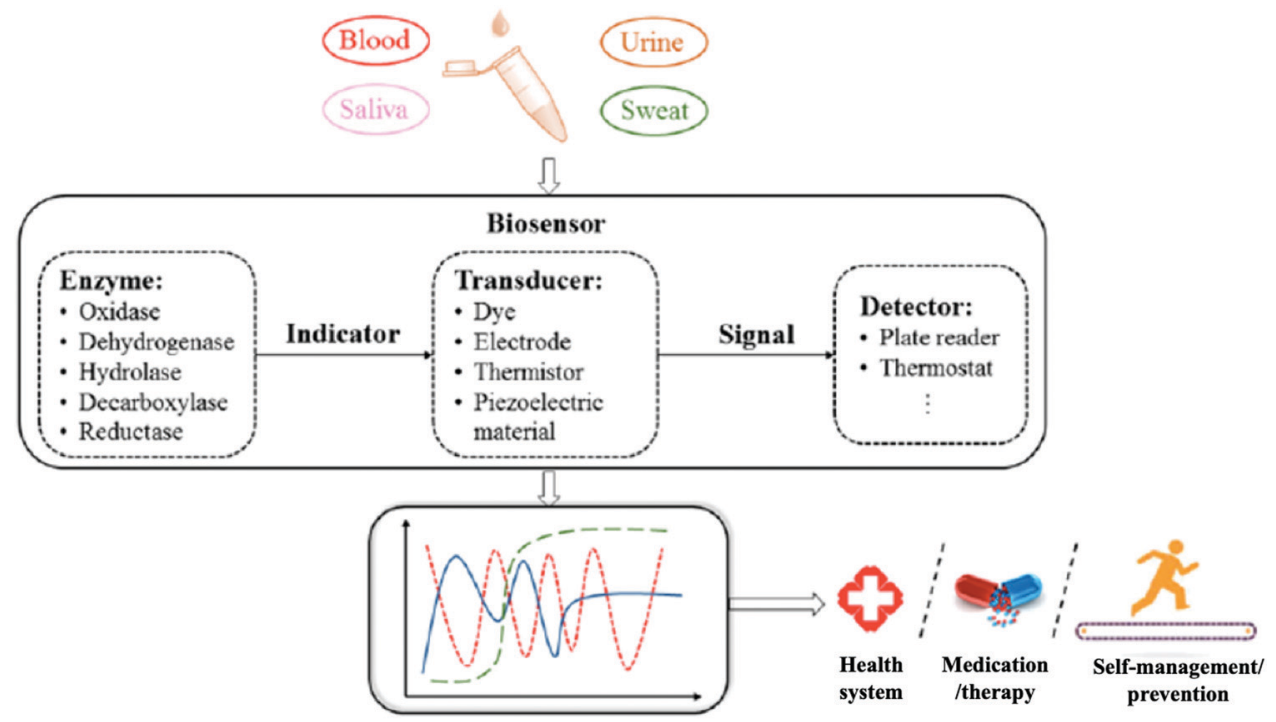

Fig. 15 A systematic procedure for the design of an enzymatic biosensor for body fluid analysis. Adapted with permission from ref. 57 . Copyright 2019 American Chemical Society. 
portable device will enable the conduct of affordable and reliable biofluid detection in rural area and developing countries.

\section{Conflicts of interest}

There are no conflicts to declare.

\section{Acknowledgements}

We acknowledge the Australia-China Science and Research Fund, Joint Research Centre on Personal Health Technologies for support.

\section{References}

1 P. J. Danneman, M. A. Suckow and C. Brayton, The laboratory mouse, CRC Press, 2012, pp. 7-10.

2 S. B. Su, T. C. W. Poon and V. Thongboonkerd, Human body fluid, BioMed Res. Int., 2013, 918793.

3 N. A. Brunzel, Fundamentals of Urine and Body Fluid AnalysisE-Book, Elsevier Health Sciences, 2016, pp. 18-26.

4 S. P. Humphrey and R. T. Williamson, A review of saliva: normal composition, flow, and function, J. Prosthet. Dent., 2001, 85, 162-169.

5 S. J. Montain, S. N. Cheuvront and H. C. Lukaski, Sweat mineral-element responses during $7 \mathrm{~h}$ of exercise-heat stress, Int. J. Sport Nutr. Exercise Metab., 2007, 17, 574-582.

6 E. Emmett, The excretion of trace metals in human sweat, Ann. Clin. Lab. Sci., 1978, 8, 270-275.

7 R. Saraymen, E. Kilic and S. Yazar, Sweat copper, zinc, iron, magnesium and chromium levels in national wrestler, Inonu Universitesi Tip Fakultesi Dergisi, 2004, 11, 7-10.

8 S. Halder, S. Samanta and G. Das, Exploring the potential of a urea derivative: an AIE-luminogen and its interaction with human serum albumin in aqueous medium, Analyst, 2019, 144, 2696-2703.

9 A. S. Levey and J. Coresh, Chronic kidney disease, Lancet, 2012, 379, 165-180.

10 Y. Hong, J. W. Lam and B. Z. Tang, Aggregation-induced emission: phenomenon, mechanism and applications, Chem. Commun., 2009, 4332-4353.

11 D. Ding, K. Li, B. Liu and B. Z. Tang, Bioprobes based on AIE fluorogens, Acc. Chem. Res., 2013, 46, 2441-2453.

12 N. Goswami, Q. Yao, Z. Luo, J. Li, T. Chen and J. Xie, Luminescent metal nanoclusters with aggregation-induced emission, J. Phys. Chem. Lett., 2016, 7, 962-975.

13 J. W. Ridley, Fundamentals of the Study of Urine and Body Fluids, Springer, 2018, pp. 1-6.

14 J. Du, Q. Gu, J. Chen, J. Fan and X. Peng, A novel fluorescent probe for the ratiometric recognition of protein based on intramolecular charge transfer, Sens. Actuators, B, 2018, 265, 204-210.

15 Y. Hong, C. Feng, Y. Yu, J. Liu, J. W. Y. Lam, K. Q. Luo and B. Z. Tang, Quantitation, visualization, and monitoring of conformational transitions of human serum albumin by a tetraphenylethene derivative with aggregation-induced emission characteristics, Anal. Chem., 2010, 82, 7035-7043.

16 P. Shen, J. Hua, H. Jin, J. Du, C. Liu, W. Yang, Q. Gao, H. Luo, Y. Liu and C. Yang, Recognition and quantification of HSA: A fluorescence probe across $\alpha$-helices of site I and site II, Sens. Actuators, B, 2017, 247, 587-594.

17 M. T. Gabr and F. C. Pigge, Rhenium tricarbonyl complexes of AIE active tetraarylethylene ligands: Tuning luminescence properties and HSA-specific binding, Dalton Trans., 2017, 46, 15040-15047.

18 J. Tong, T. Hu, A. Qin, J. Z. Sun and B. Z. Tang, Deciphering the binding behaviours of BSA using ionic AIE-active fluorescent probes, Faraday Discuss., 2017, 196, 285-303.

19 R. S. Singh, A. Kumar, S. Mukhopadhyay, G. Sharma, B. Koch and D. S. Pandey, An Unconventional Mechanistic Insight on Aggregation Induced Emission in Novel Boron Dipyrromethenes and Their Rational Biological Realizations, J. Phys. Chem. C, 2016, 120, 22605-22614.

20 T. Gao, S. Yang, X. Cao, J. Dong, N. Zhao, P. Ge, W. Zeng and Z. Cheng, Smart self-assembled organic nanoprobe for protein-specific detection: design, synthesis, application, and mechanism studies, Anal. Chem., 2017, 89, 10085-10093.

21 N. Dey, B. Maji and S. Bhattacharya, Motion-Induced Changes in Emission as an Effective Strategy for the Ratiometric Probing of Human Serum Albumin and Trypsin in Biological Fluids, Chem. - Asian J., 2018, 13, 664-671.

22 Y. Tu, Y. Yu, Z. Zhou, S. Xie, B. Yao, S. Guan, B. Situ, Y. Liu, R. T. Kwok, J. W. Lam and S. Chen, Specific and quantitative detection of albumin in biological fluids by tetrazolatefunctionalized water-soluble AIEgens, ACS Appl. Mater. Interfaces, 2019, 11, 29619-29629.

23 T. Chen, N. Xie, L. Viglianti, Y. Zhou, H. Tan, B. Z. Tang and Y. Tang, Quantitative urinalysis using aggregation-induced emission bioprobes for monitoring chronic kidney disease, Faraday Discuss., 2017, 196, 351-362.

24 R. Jalili and A. Khataee, Aluminum(III) triggered aggregationinduced emission of glutathione-capped copper nanoclusters as a fluorescent probe for creatinine, Microchim. Acta, 2019, 186, 29.

25 Y. Hang, J. Wang, T. Jiang, N. Lu and J. Hua, Diketopyrrolopyrrole-based ratiometric/turn-on fluorescent chemosensors for citrate detection in the near-infrared region by an aggregation-induced emission mechanism, Anal. Chem., 2016, 88, 1696-1703.

26 C. Liu, Y. Hang, T. Jiang, J. Yang, X. Zhang and J. Hua, A light-up fluorescent probe for citrate detection based on bispyridinum amides with aggregation-induced emission feature, Talanta, 2018, 178, 847-853.

27 T. Jiang, N. Lu, Y. Hang, J. Yang, J. Mei, J. Wang, J. Hua and H. Tian, Dimethoxy triarylamine-derived terpyridine-zinc complex: a fluorescence light-up sensor for citrate detection based on aggregation-induced emission, J. Mater. Chem. C, 2016, 4, 10040-10046.

28 X. Li, Z. Zhou, Y. Tang, C. C. Zhang, Y. Zheng, J. Gao and Q. Wang, Modulation of assembly and disassembly of a new tetraphenylethene based nanosensor for highly selective detection of hyaluronidase, Sens. Actuators, B, 2018, 276, 95-100. 
$29 \mathrm{H}$. Xie, F. Zeng and S. Wu, Ratiometric fluorescent biosensor for hyaluronidase with hyaluronan as both nanoparticle scaffold and substrate for enzymatic reaction, Biomacromolecules, 2014, 15, 3383-3389.

30 S. K. Kim, D. H. Lee, J. I. Hong and J. Yoon, Chemosensors for pyrophosphate, Acc. Chem. Res., 2009, 42, 23-31.

31 M. Doherty, C. Belcher, M. Regan, A. Jones and J. Ledingham, Association between synovial fluid levels of inorganic pyrophosphate and short term radiographic outcome of knee osteoarthritis, Ann. Rheum. Dis., 1996, 55, 432-436.

32 C. Li, Y. Chen, L. Yang, C. Zhang, B. Wang and Y. Wang, Tetraphenylethene decorated hyperbranched poly (amido amine) s as metal/organic-solvent-free turn-on AIE probe for specific pyrophosphate detection, Sens. Actuators, B, 2019, 291, 25-33.

33 Q. Wang, X. Wen and Z. Fan, A Schiff base fluorescent chemsensor for the double detection of $\mathrm{Al} 3+$ and PPi through aggregation induced emission in environmental physiology, J. Photochem. Photobiol., A, 2018, 358, 92-99.

34 R. Rajamanikandan and M. Ilanchelian, Highly selective and sensitive biosensing of dopamine based on glutathione coated silver nanoclusters enhanced fluorescence, New J. Chem., 2017, 41, 15244-15250.

35 S. Ghosh, J. R. Bhamore, N. I. Malek, Z. V. P. Murthy and S. K. Kailasa, Trypsin mediated one-pot reaction for the synthesis of red fluorescent gold nanoclusters: Sensing of multiple analytes (carbidopa, dopamine, $\mathrm{Cu} 2+$, $\mathrm{Co} 2+$ and Hg2+ ions), Spectrochim. Acta, Part A, 2019, 215, 209-217.

36 R. Singh, N. Gautam, A. Mishra and R. Gupta, Heavy metals and living systems: An overview, Indian J. Pharmacol., 2011, 43, 246.

37 M. Jaishankar, T. Tseten, N. Anbalagan, B. B. Mathew and K. N. Beeregowda, Toxicity, mechanism and health effects of some heavy metals, Interdiscip. Toxicol., 2014, 7, 60-72.

38 R. X. Zhang, P. F. Li, W. J. Zhang, N. Li and N. Zhao, A highly sensitive fluorescent sensor with aggregation-induced emission characteristics for the detection of iodide and mercury ions in aqueous solution, J. Mater. Chem. C, 2016, 4, 10479-10485.

39 X. Wen, Q. Wang and Z. Fan, Highly selective turn-on fluorogenic chemosensor for $\mathrm{Zn}$ (II) detection based on aggregation-induced emission, J. Lumin., 2018, 194, 366-373.

40 L. Zhao, T. Wang, Q. Wu, Y. Liu, Z. Chen and X. Li, Fluorescent strips of electrospun fibers for ratiometric sensing of serum heparin and urine trypsin, ACS Appl. Mater. Interfaces, 2017, 9, 3400-3410.

41 Y. Liu, C. Deng, L. Tang, A. Qin, R. Hu, J. Z. Sun and B. Z. Tang, Specific detection of D-glucose by a tetraphenylethenebased fluorescent sensor, J. Am. Chem. Soc., 2010, 133, 660-663.

42 G. Jiang, J. Wang, Y. Yang, G. Zhang, Y. Liu, H. Lin, G. Zhang, Y. Li and X. Fan, Fluorescent turn-on sensing of bacterial lipopolysaccharide in artificial urine sample with sensitivity down to nanomolar by tetraphenylethylene based aggregation induced emission molecule, Biosens. Bioelectron., 2016, 85, 62-67.

43 J. A. Rathmacher, J. C. Fuller, S. M. Baier, N. N. Abumrad, H. F. Angus and R. L. Sharp, Adenosine-5'-triphosphate
(ATP) supplementation improves low peak muscle torque and torque fatigue during repeated high intensity exercise sets, J. Int. Soc. Sports Nutr., 2012, 9, 48.

44 X. Liu, Y. Yu, B. Lin, Y. Cao and M. Guo, A labelfree fluorescent probe for the detection of adenosine 5 'triphosphate via inhibiting the aggregation-induced emission enhancement of glutathione modified silver nanoclusters triggered by zinc ion, Spectrochim. Acta, Part A, 2019, 214, 360-365.

45 Y. Hu, J. Liu, X. You, C. Wang, Z. Li and W. Xie, A light-up probe for detection of adenosine in urine samples by a combination of an AIE molecule and an aptamer, Sensors, 2017, 17, 2246.

46 G. Jiang, W. Zhu, Q. Chen, X. Li, G. Zhang, Y. Li, X. Fan and J. Wang, Selective fluorescent probes for spermine and 1-adamantanamine based on the supramolecular structure formed between AIE-active molecule and cucurbit [n] urils, Sens. Actuators, B, 2018, 261, 602-607.

47 X. Zhang, R. Kong, Q. Tan, F. Qu and F. Qu, A label-free fluorescence turn-on assay for glutathione detection by using $\mathrm{MnO} 2$ nanosheets assisted aggregation-induced emission-silica nanospheres, Talanta, 2017, 169, 1-7.

48 L. Yu, H. Chen, J. Yue, X. Chen, M. Sun, H. Tan, A. M. Asiri, K. A. Alamry, X. Wang and S. Wang, Metal-Organic Framework Enhances Aggregation-Induced Fluorescence of Chlortetracycline and the Application for Detection, Anal. Chem., 2019, 91, 5913-5921.

49 Y. Zhang, Z. Zhou, J. Zheng, H. Li, J. Cui, S. Liu, Y. Yan and C. Li, SiO 2-MIP core-shell nanoparticles containing gold nanoclusters for sensitive fluorescence detection of the antibiotic erythromycin, Microchim. Acta, 2017, 184, 2241-2248.

50 Z. Zhang, R. T. Kwok, Y. Yu, B. Z. Tang and K. M. Ng, Sensitive and specific detection of L-lactate using an AIEactive fluorophore, ACS Appl. Mater. Interfaces, 2017, 9, 38153-38158.

51 Z. An, S. Chen, X. Tong, H. He, J. Han, M. Ma, Y. Shi and X. Wang, Widely Applicable AIE Chemosensor for On-Site Fast Detection of Drugs Based on the POSS-Core Dendrimer with the Controlled Self-Assembly Mechanism, Langmuir, 2019, 35, 2649-2654.

52 F. Bu, B. Zhao, W. Kan, L. Wang, B. Song, J. Wang, Z. Zhang, Q. Deng and G. Yin, A phenanthro [9, 10-d] imidazole-based AIE active fluorescence probe for sequential detection of $\mathrm{Ag}$ +/AgNPs and SCN- in water and saliva samples and its application in living cells, Spectrochim. Acta, Part A, 2019, 223, 117333.

53 N. De Giovanni and N. Fucci, The current status of sweat testing for drugs of abuse: A review, Curr. Med. Chem., 2013, 20, 545-561.

54 A. J. Bandodkar, V. W. Hung, W. Jia, G. Valdés-Ramírez, J. R. Windmiller, A. G. Martinez, J. Ramírez, G. Chan, K. Kerman and J. Wang, Tattoo-based potentiometric ionselective sensors for epidermal pH monitoring, Analyst, 2013, 138, 123-128. 
55 S. Jadoon, S. Karim, M. R. Akram, A. Kalsoom Khan, M. A. Zia, A. R. Siddiqi and G. Murtaza, Recent developments in sweat analysis and its applications, Int. J. Anal. Chem., 2015, 2015, 164974.

56 J. Heikenfeld, Non-invasive analyte access and sensing through eccrine sweat: Challenges and outlook circa 2016, Electroanalysis, 2016, 28, 1242-1249.
57 Z. Zhang, X. Zhang, K. Y. Fung and K. M. Ng, Product Design: Enzymatic Biosensors for Body Fluid Analysis, Ind. Eng. Chem. Res., 2019, 58, 14284-14294.

58 S. Akraa, A. P. T. Tam, H. Shen, Y. Tang, B. Z. Tang, J. Li and S. Walker, A smartphone-based point-of-care quantitative urinalysis device for chronic kidney disease patients, J. Netw. Comput. Appl., 2018, 115, 59-69. 